\title{
Particle in a cavity in one-dimensional bandlimited quantum mechanics
}

\author{
K. Sailer, ${ }^{1}$ Z. Péli, ${ }^{1}$ and S. Nagy ${ }^{1}$ \\ ${ }^{1}$ Department of Theoretical Physics, University of Debrecen, P.O. Box 5, H-4010 Debrecen, Hungary
}

(Dated: August 29, 2018)

\begin{abstract}
The effects of the generalized uncertainty principle (GUP) on the low-energy stationary states of a particle moving in a cavity with no sharp boundaries are determined by means of the perturbation expansion in the framework of one-dimensional bandlimited quantum mechanics. A realization of GUP resulting in the existence of a finite ultraviolet (UV) wave-vector cutoff $K \sim 1 / \ell_{P}$ (with the Planck length $\left.\ell_{P}\right)$ is considered. The cavity of the size $\ell \gg \ell_{P}$ is represented by an infinitely deep trapezoid-well potential with boundaries smeared out in a range $R$ satisfying the inequalities $\ell \gg R \gtrsim \ell_{P}$. In order to determine the energy shifts of the low-lying stationary states, the usual perturbation expansion is reformulated in a manner that enables one to treat consistently orderby-order the direct and indirect GUP effects, i.e., those due to the modification of the Hamiltonian and the lack of the UV modes, respectively. It is shown that the leading terms of the indirect and the direct GUP effects are of the first and second order, respectively, in the small parameter $\ell_{P} / \ell$ in agreement with our previous finding in a more naive approach [1].
\end{abstract}

PACS numbers: $04.60 . \mathrm{Bc}$

\section{INTRODUCTION}

Here our goal is to determine the stationary states of a particle in a cavity in the framework of the onedimensional bandlimited quantum mechanics. This issue is a reconsideration of the 'particle in the box' problem, but now we remove the sharp boundaries of the cavity describing it by a trapezoid-well potential of infinite depth. Furthermore, we implement the nondegenerate stationary perturbation expansion in a manner that enables us to distinguish of the various orders of the direct and indirect GUP effects. In this way we shall recover our basic finding in [1] in a more reliable framework, namely, that the indirect GUP effect is much more important than the direct GUP effect as to the energy shifts of the low-lying stationary states.

In the last two decades there has been a continuous interest in effective quantum mechanics based on GUP [2-9], the various modifications of Heisenberg's uncertainty principle, that is motivated by theoretical indications [10-13] pointing towards the consequences quantum gravity may have on the behaviour of low-energy quantum systems. Among the various realizations of GUP there is a class when the deformation of the commutator relation for the operators of the coordinate $\hat{x}$ and the canonical momentum $\hat{p}_{x}$ depends only on the canonical momentum,

$$
\left[\hat{x}, \hat{p}_{x}\right]=i \hbar f\left(\alpha\left|\hat{p}_{x}\right|\right)
$$

with the deformation function $f(|u|)$, where $u=\alpha p_{x}$ and $\alpha=\mathcal{O}(a / \hbar)$ with some minimal length scale $a$, a kind of the quantum of distance. Here we shall use the widespread conjecture that $a$ is equal to the Planck length $a=\ell_{P} \approx 1.616 \times 10^{-35} \mathrm{~m}$, although an unquestionable proof for that does not exist [8]. Therefore there is an alternative view point that the minimal length scale $a$ must be determined from experimental data and various upper bounds on it have been given [14-23]. In the present paper we shall restrict ourselves to the particular form of the deformation function $f=1+\alpha^{2} p_{x}^{2}$ for which there exists a minimal wavelength, i.e., a maximal magnitude $K=\pi / a$ of the wave vector, but the canonical momentum $p_{x}=\alpha^{-1} \tan \left(\alpha \hbar k_{x}\right)$ can take arbitrarily large values $[24,25]$. Then the physical states are restricted to those of finite bandwidth, i.e., the wave-vector operator $\hat{k}_{x}=(\alpha \hbar)^{-1} \arctan \left(\alpha \hat{p}_{x}\right)$ can only take eigenvalues in the interval $[-K, K]$.

In the present paper we shall replace the infinitely deep square-well potential, generally used in the discussions of the 'particle in the box' problem, with a potential with smeared out boundaries. It was noticed already in [26] that the usage of a potential with sharp boundaries is in contradiction with the existence of the minimal length scale $a$ in bandlimited quantum mechanics. Space has particular features in one-dimensional bandlimited quantum mechanics [27]. Although the wave-vector and the canonical momentum operators are self-adjoint, the coordinate operator $\hat{x}$ is only Hermitian symmetric. It has, however, a one-parameter family of self-adjoint extensions with eigenvalues determining a grid with equidistant spacing on the coordinate axis, defining a minimal length scale $a$, while the grids belonging to the various extensions can be continuously shifted into each other. This is a sign that positions can only be observed with a maximal precision $\Delta x_{\min } \approx a$, while momenta can be measured with arbitrary accuracy [6, 24, 28-33]. There exist formal coordinate eigenstates, representing the eigenvectors of any of the self-adjoint extensions of the coordinate operator, but those cannot be approximated now by a sequence of physical states with uncertainties in position decreasing to zero [24]. Nevertheless, they form an appropriate basis to decompose any physical state into a linear superposition of coordinate eigenstates. In our previous paper [1] we discussed in detail that such a decomposition of any physical state corresponds to the generalization of Shannon's sampling theorem [34]. In bandlimited quan- 
tum mechanics the coordinate space turns out to exhibit features of discreteness [35] and continuity at the same time like information does [36-42]. The main idea is that space can be thought of a differentiable manifold, but the physical degrees of freedom cannot fill it in arbitrarily dense manner. It has also been conjectured that degrees of freedom corresponding to structures smaller than the resolvable Planck scale turn into internal degrees of freedom [43-46]. The square-integrable coordinate wavefunctions do not have the usual probabilistic meaning, yet provide useful tools to characterize the quantum states of the particle which can then be analyzed e.g. in terms of maximally localized states [28-31].

Keeping in mind the above described structure of space, now we choose an infinitely deep trapezoid-well potential to model the cavity. Then the boundaries of the cavity are smeared out in intervals of the size $2 R$, where $R$ is much less than the size $\ell$ of the cavity. It is assumed that the inaccuracy $\sim R$ of the determination of the positions of the boundaries should be of the order of the minimal length scale if that inaccuracy is caused solely by the space structure at the Planckian length scale. In other terms it is assumed that no many-body physics of the medium surrounding the cavity can provide boundaries more sharp than it is allowed by the existence of the minimal length scale. In realistic cases, when the cavity is physically prepared in a medium and is of the size on nanoscales, the unsharpness of its boundaries is basicly determined by some interactions of range much larger than the Planck scale, but even then can hold the inequality $R \ll \ell$. The result obtained below shall be valid for any values of the parameter $R$ for which the inequalities $\ell \gg R \gtrsim a$ hold. The grid of the coordinate eigenvalues seems to dictate that the distances $\ell$ and $R$ have to be considered as integer times the spacing $a$.

In order to determine the low-energy spectrum of the particle in the cavity we shall solve the Schrödinger equation for the coordinate wavefunctions in the case when GUP implies finite bandwidth. It is well-known that GUP directly affects the Hamiltonian through the modification of the canonical momentum and that of the kinetic energy operator $\left(\hat{p}_{x}^{2} / 2 m\right)-\left(\hbar^{2} \hat{k}_{x}^{2} / 2 m\right)$ which can be expanded - when low-energy states are considered in the powers of the small parameter $\alpha k \sim a / \ell$ where $k$ is the characteristic wave vector of the given stationary state. This direct GUP effect has been treated in the framework of the perturbation expansion using the coordinate representation and discussed in detail for various quantum systems (see Refs. [25, 47-67] without the quest of completeness), among others for the particle in a box $[47,48,54,55,58,62,63,68]$. In that connection it was discussed the problem of prescribing boundary conditions $[69,70]$ and the existence of the self-adjoint extensions of various forms of the GUP modified Hamiltonian [71]. Generally the perturbative discussions of the various low-energy quantum systems were performed either for realizations of GUP when no wave-vector cutoff $K$ occurs or with the neglection of the indirect GUP ef- fect caused by such an UV wave-vector cutoff. One were inclined to think that the existence of a very high UV cutoff, $K \sim \pi / a \sim 10^{35} \mathrm{~m}^{-1}$ has negligible effect on the low-energy states of the quantum system. Nevertheless, in our previous paper [1] it was shown on the example of the 'particle in the box' problem that the indirect GUP effect may be even much more important than the direct GUP effect is. The energy shifts of the low-energy stationary states of a particle in the infinitely deep square-well potential were determined in the framework of the bandlimited quantum mechanics considering the latter as an effective theory in which no quantum fluctuations of wavelength smaller than those of the order of the minimal length are possible, i.e., in which the coordinate wavefunctions should not contain Fourier components with wave vectors outside of the finite band $k_{x} \in[-K, K]$. In order to built in this restriction into the Schrödinger equation we developed a projection technique that essentially means that the wavefunctions and the operators are projected by means of the projector $\hat{\Pi}$ onto the bandlimited subspace $\mathcal{H}$ of the Hilbert space. We have seen that in the case of the stationary states of the particle in the box, i.e., in a square-well potential of infinite depth the indirect GUP effect resulting form the existence of the finite bandwidth is an effect of the first order, while for a nonrelativistic particle the direct GUP effect is an effect of the second order in the small parameter $a / \ell$. Motivated by this finding we shall develop here a perturbation expansion up to the second order making the working hypothesis that the indirect and the direct GUP effects are of the first and second orders, respectively. We shall show that the consistent separation of the various orders in the small parameter $a / \ell$ becomes possible in the case of infinitely deep trapezoid-well potential that justifies a posteriori our working hypothesis. In this manner we can confirm our previous result in a more reliable framework.

A similar result, but in somewhat other terms was found for the particle in a box in Ref. [26] where it was realized that the model with precisely given box size $\ell$ is ill-defined in the sense that a change of the box size of the order $\ell_{P}$, i.e., that of the maximal accuracy $\Delta x_{\min }$ of the position determination causes an energy shift of the order $\mathcal{O}\left(\ell_{P} / \ell\right)$ as compared to the direct GUP effect of the order $\mathcal{O}\left(\left(\ell_{P} / \ell\right)^{2}\right)$. Here we improve our previous approach to the 'particle in box' problem in [1] by (i) replacing the box, the infinitely deep square-well potential with sharp boundaries by an infinitely deep trapezoidwell potential with smeared out boundaries (ii) and by an order-by-order consistent usage of the perturbation expansion. The main message, the dominance of the indirect GUP effect over the direct one has been recovered. The sharp boundaries of the box potential caused periodic dependence of the relative energy shifts of the low-energy stationary states on the ratio $\ell / a[1]$. Now we shall see that a similar periodic dependence on the ratio $(\ell-2 R) / a$ remains still present after the smearing out the boundaries of the cavity in the range $2 R$ when 
the trapezoid-well potential is used, since the 'effective length' of the cavity equals now to $\ell-2 R$.

Our paper is constructed as follows. In Section II the perturbation Hamiltonian and the usage of the nondegenerate stationary perturbation expansion are given. In Section III we apply the perturbation expansion to the determination of the stationary states of the particle in the cavity modelled by an infinitely deep trapezoid-well potential and discuss the results obtained for the relative energy shifts of the low-energy states due to the GUP effect. A short summary is given in Section IV. In Appendix A the unperturbed Schrödinger equation is solved for the particle in the trapezoid-well potential keeping the depth of the potential finite but much larger than the particle's energy. A reminder for the derivation of the explicit formulas of the nondegenerate stationary perturbation expansion is given in Appendix B for the case when the perturbation Hamiltonian itself consists of various order terms. Finally, the evaluation and estimation of the matrix elements of the various terms of the perturbation Hamiltonian are given in Appendix C.

\section{PERTURBATION EXPANSION}

We describe the cavity by the trapezoid-well potential parametrized as

$$
\begin{aligned}
& V_{t}(x) \\
& =V_{\infty} \begin{cases}1 & \text { for }(\ell / 2)+R \leq|x| \\
\frac{R+|x|-(\ell / 2)}{2 R} & \text { for }(\ell / 2)-R \leq|x|<(\ell / 2)+R \\
0 & \text { for } 0 \leq|x|<(\ell / 2)-R\end{cases}
\end{aligned}
$$

The potential $V_{t}(x)$ is symmetric, $V_{t}(x)=V_{t}(-x)$, takes the constant value $V_{\infty}$ asymptotically far away from the origin, and decreases to and increases from zero linearly at the boundaries of the cavity at $x=-\ell / 2$ and $\ell / 2$, respectively, in an interval of the length $2 R \ll \ell$. In this way the boundaries of the cavity are smeared out on a distance $2 R$. In the limit $V_{\infty} \rightarrow \infty$ the potential becomes infinitely deep and formally it goes over into a square-well potential of the size $\ell-2 R$. The limit is, however, not smooth, because for any finite but large values of $V_{\infty}$ the boundary regions of the potential-well still keep their size. Therefore, the quantum mechanical expectation values of some observables of the particle in stationary states may become different for an infinitely deep trapezoid-well potential and for an infinitely deep square-well potential. We shall perform the calculations for a trapezoid-well potential with finite but large values of $V_{\infty}$ and take the limit $V_{\infty} \rightarrow \infty$ when the energy eigenvalues have already been evaluated. We shall see that the results obtained for the relative energy shifts of the stationary states differ somewhat from those obtained in our previous work [1]. One has to notice that a potential imitating a cavity of the size $\approx \ell$ with obscure boundaries can be chosen in infinitely many ways.
Our choice is simple enough enabling us to find analytic solution of the unperturbed Schrödinger equation.

In the bandlimited quantum mechanics the stationary Schrödinger-equation,

$$
\hat{H} \psi_{\nu}=\varepsilon_{\nu} \psi_{\nu}
$$

for the particle of mass $m$ in the cavity represented by the trapezoid-well potential (2) is given in terms of the Hamiltonian with the kernel

$$
H(x, y)=T_{\Pi}(x, y)+V_{\Pi}(x, y),
$$

where

$$
T_{\Pi}(x, y)=\int_{-\infty}^{\infty} d z \Pi(x-z) \frac{\left[\tan \left(-i \alpha \hbar \partial_{z}\right)\right]^{2}}{2 m \alpha^{2}} \Pi(z-y)
$$

and

$$
V_{\Pi}(x, y)=\int_{-\infty}^{\infty} d z \Pi(x-z) V_{t}(z) \Pi(z-y)
$$

are the kernels of the projected kinetic and potential energy operators, respectively, and the kernel of the projector $\hat{\Pi}$ is given as

$$
\Pi(x-y)=\int_{-K}^{K} \frac{d k_{x}}{2 \pi} e^{i k_{x}(x-y)}=\frac{\sin [K(x-y)]}{\pi(x-y)}
$$

(see [1]). Instead of solving the stationary Schrödingerequation (3) directly, we shall determine the stationary states by means of the perturbation expansion. The Hamiltonian kernel of the unperturbed system is now chosen as

$$
H_{0}(x, y)=\left(-\frac{\hbar^{2}}{2 m} \partial_{x}^{2}+V_{t}(x)\right) \delta(x-y)
$$

Then the solution of the stationary Schrödinger-equation,

$$
\hat{H}_{0} \phi_{\nu}=\epsilon_{\nu} \phi_{\nu}
$$

i.e., the determination of the stationary states of the unperturbed system is a problem stated in the framework of ordinary quantum mechanics and can be solved analytically (see Appendix A). The choice (8) of the unperturbed Hamiltonian reflects our working hypothesis that both the direct GUP effect, i.e., the effect due to the modification of the kinetic energy operator, as well as the indirect GUP effect occurring due to the projection to the subspace of bandlimited wavefunctions can be considered as perturbations. Such an assumption is motivated by previous findings for the low-energy stationary states of a nonrelativistic particle in a box, i.e., in an infinitely deep square-well potential exhibiting sharp boundaries. On the one hand, it is well-known that the direct GUP effect is of the order $(a / \ell)^{2}$ for a nonrelativistic particle in a box $[47,48,54,55,58,62,63]$ and, on the other hand, we have found that the indirect GUP effect is even more important but still perturbative and of the order $a / \ell[1]$. In the latter case we did not, however, 
made a distinction between the contributions of the various orders in the small parameter $a / \ell$. Our purpose is now to show in the framework of a more consistent perturbative treatment that the indirect GUP effect caused by the lack of UV wave-vector modes is perturbative and of the order $a / \ell$ indeed. Moreover, with the choice of the trapezoid-well potential of infinite depth instead of the square-well potential with sharp boundaries, we would like to remove the inconsistency pointed out in [26].

Ordinary quantum mechanics for a particle in a box of size $\ell$ can be used to estimate the low-energy part of the spectrum as $\epsilon_{\nu}^{b o x}=\frac{\hbar^{2} \pi^{2}(\nu+1)^{2}}{2 m \ell^{2}}=\frac{\hbar\left(k_{\nu}^{b o x}\right)^{2}}{2 m}$ with the wave vector $k_{\nu}^{b o x}=\frac{\pi(\nu+1)}{\ell}$ with $\nu=0,1,2, \ldots$. We expect that the energies $\epsilon_{\nu}$ and $\varepsilon_{\nu}$ are close to the values $\epsilon_{\nu}^{b o x}$ in the low-energy part of the spectrum when the depth of the potential well $V_{\infty}$ is chosen to be much larger than $\epsilon_{0}^{b o x}$. Finally, we shall take the limit $V_{0} \rightarrow \infty$ and that enables us to separate the various orders of the perturbation expansion in a rather straightforward manner.

As compared to the unperturbed Hamiltonian $\hat{H}_{0}$ the direct GUP effect results in the deviation $\hat{h}_{t}=\left(\hat{p}_{x}^{2}-\right.$ $\left.\hbar^{2} \hat{k}_{x}^{2}\right) /(2 m)$ of the GUP modified kinetic energy operator from the ordinary one. The indirect GUP effects arise from the modification $\hat{t}=\hat{T}_{\Pi}-\hat{T}$ and $\hat{v}=\hat{V}_{t \Pi}-\hat{V}_{t}$ of the kinetic and potential energy operators, respectively due to projection onto the bandlimited subspace. Then the Hamiltonian of the perturbation, $\delta \hat{H}=\hat{H}-\hat{H}_{0}$ can be rewritten as

$$
\delta \hat{H}=\hat{h}_{t}+\hat{t}+\hat{v}
$$

with the kernels

$$
\begin{aligned}
h_{t}(x, y) & =\frac{1}{2 m}\left(\alpha^{-2} \tan ^{2}\left(-i \alpha \hbar \partial_{x}\right)-\hbar^{2} \partial_{x}^{2}\right) \delta(x-y), \\
t(x, y) & =\frac{1}{2 m \alpha^{2}} \tan ^{2}\left(-i \alpha \hbar \partial_{x}\right)[\Pi(x-y)-\delta(x-y)], \\
v(x, y) & =\frac{1}{2}\left[V_{t}(x)+V_{t}(y)\right][\Pi(x-y)-\delta(x-y)], \quad(11)
\end{aligned}
$$

where we applied the rules established in [1] to write down the kernels of the projected operators in the framework of bandlimited quantum mechanics. The usual nondegenerate stationary perturbation expansion provides the corrections of the various orders to the energy levels and the corresponding wavefunctions in terms of the matrix elements $\left\langle\phi_{\nu}|\delta \hat{H}| \phi_{\nu^{\prime}}\right\rangle$ of the perturbation Hamiltonian $\delta \hat{H}$ sandwiched by the unperturbed stationary wavefunctions $\phi_{\nu}$ and $\phi_{\nu^{\prime}}$. Now we shall apply this perturbation scheme assuming that the matrix elements of the perturbation Hamiltonian can be recasted into the sum of those of perturbation operators $\delta^{[n]} \hat{H}$ of various orders $n$ in the small parameter $a / \ell$, for which

$$
\delta \hat{H}=\sum_{n=1}^{\infty} \delta^{[n]} \hat{H}
$$

holds. We shall show that in the limit $V_{0} \rightarrow \infty$ it is possible to identify the pieces of various orders of the matrix elements $\left\langle\phi_{\nu}|\delta \hat{H}| \phi_{\nu^{\prime}}\right\rangle$, i.e., those of the matrix elements of the operators $\hat{h}_{t}, \hat{t}$, and $\hat{v}$.

As compared to the unperturbed Hamiltonian $\hat{H}_{0}$ the direct GUP effect results in the additional terms $\hat{h}_{t}=$ $\sum_{n=1}^{\infty} \hat{h}_{t}^{(n)}$ modifying the ordinary kinetic energy operator, where $\hat{h}_{t}^{(n)} \sim \mathcal{O}\left((a / \ell)^{n}\right)$. For the deformation function $f\left(p_{x}\right)=1+\alpha^{2} p_{x}^{2}$ the expansions $p_{x}=\tan u \approx$ $u+\frac{u^{3}}{3}+\ldots$ with $u=\hbar k_{x}$ and $\tan ^{2} u \approx u^{2}+\frac{2 u^{4}}{3}=$ $u^{2}\left(1+\frac{2}{3} u^{2}\right)$ imply the kernels $h_{t}^{[1]}(x, y)=0$ and

$$
h_{t}^{[2]}(x, y)=\frac{2 \alpha^{2}}{3} \frac{\hbar^{4}}{2 m} \partial_{x}^{4} \delta(x-y) .
$$

Similarly the piece $\hat{t}$ of the perturbation Hamiltonian associated with the projection of the kinetic energy operator can be split as $\hat{t}=\hat{t}^{[1]}+\hat{t}^{[2]}+\ldots$ with the kernels

$$
\begin{aligned}
t^{[1]}(x, y) & =-\frac{\hbar^{2}}{2 m} \partial_{x}^{2}[\Pi(x-y)-\delta(x-y)], \\
t^{[2]}(x, y) & =\frac{2 \alpha^{2}}{3} \frac{\hbar^{4}}{2 m} \partial_{x}^{4}[\Pi(x-y)-\delta(x-y)],
\end{aligned}
$$

where the presence of the projector $\hat{1}-\hat{\Pi}$ onto the subspace of the UV modes is treated as the one causing a first-order effect. In the same line also the perturbation $\hat{v}$ with the kernel

$$
v^{[1]}(x, y)=\frac{1}{2}\left[V_{t}(x)+V_{t}(y)\right][\Pi(x-y)-\delta(x-y)](16)
$$

shall be treated as that of the first order. At the end of the evaluation of the matrix elements of these operators between the unperturbed states $\phi_{\nu}$ we shall see that they contain terms of higher orders too. This seeming inconsistency can however be cured by reshuffling the appropriate second order terms from the matrix elements $\left\langle\phi_{\nu}\left|\delta \hat{H}^{[1]}\right| \phi_{\nu^{\prime}}\right\rangle$ into the matrix elements $\left\langle\phi_{\nu}\left|\delta \hat{H}^{[2]}\right| \phi_{\nu^{\prime}}\right\rangle$ and neglecting all contributions of higher than second order when we restrict ourselves to the second order of the perturbation expansion, at which the direct GUP effect comes into play. Therefore, the selection of the terms of various orders made above on the level of operators has to be reconsidered and made more accurate at the level of the matrix elements. This happens because the projector introduces $a$-dependence into the kernels of the operators that cannot be Taylor-expanded at the operator level. We shall see that for the trapezoid-well potential of infinite depth the separation of the various orders at the level of the matrix elements is straightforward. It should be noted that the possibility to treat the indirect GUP effect as perturbation seems to be a particular feature of the case when the particle moves in a potential of infinite depth. For a potential of finite depth there would occur nonanalytic dependences of the matrix elements on the length scale $a$ through the explicit dependence of the projector (7) on the wave-vector cutoff $K=\pi / a$ (see e.g. our remark at the end of the paragraph following Eq. (C28) in Appendix C). This makes questionable the 
perturbative treatment of the indirect GUP effect in the case of a potential well of finite depth.

Now assuming that we keep the terms of various orders of the perturbation Hamiltonian under control, we insert its expansion (12) together with the expansion of the energy eigenvalue and that of the wavefunction,

$$
\begin{aligned}
& \varepsilon_{\nu}=\epsilon_{\nu}+\sum_{n=1}^{\infty} \delta^{[n]} \epsilon_{\nu}, \\
& \psi_{\nu}=\phi_{\nu}+\sum_{n=1}^{\infty} \delta^{[n]} \psi_{\nu},
\end{aligned}
$$

respectively, into the stationary Schrödinger equation (3) and repeat the steps being a piece of textbook material on nondegenerate perturbation expansion in ordinary quantum mechanics (for a reminder see Appendix B). This yields then for the corrections of the first order

$$
\begin{aligned}
\delta^{[1]} \epsilon_{\nu} & =\left\langle\phi_{\nu}\left|\delta^{[1]} \hat{H}\right| \phi_{\nu}\right\rangle \\
\delta^{[1]} \psi_{\nu} & =-\sum_{\mu \neq \nu} \frac{\left\langle\phi_{\mu}\left|\delta^{[1]} \hat{H}\right| \phi_{\nu}\right\rangle}{\epsilon_{\mu}-\epsilon_{\nu}} \phi_{\mu},
\end{aligned}
$$

and those of the second order,

$$
\begin{aligned}
\delta^{[2]} \epsilon_{\nu}= & \left\langle\phi_{\nu}\left|\delta^{[2]} \hat{H}\right| \phi_{\nu}\right\rangle-\sum_{\mu \neq \nu} \frac{\left|\left\langle\phi_{\mu}\left|\delta^{[1]} \hat{H}\right| \phi_{\nu}\right\rangle\right|^{2}}{\epsilon_{\mu}-\epsilon_{\nu}}, \\
\delta^{[2]} \psi_{\nu}= & -\sum_{\mu \neq \nu} \frac{\left\langle\phi_{\mu}\left|\delta^{[2]} \hat{H}\right| \phi_{\nu}\right\rangle}{\epsilon_{\mu}-\epsilon_{\nu}} \phi_{\mu} \\
& +\sum_{\mu, \mu^{\prime} \neq \nu} \frac{\left\langle\phi_{\mu}\left|\delta^{[1]} \hat{H}\right| \phi_{\mu^{\prime}}\right\rangle\left\langle\phi_{\mu^{\prime}}\left|\delta^{[1]} \hat{H}\right| \phi_{\nu}\right\rangle}{\left(\epsilon_{\mu}-\epsilon_{\nu}\right)\left(\epsilon_{\mu^{\prime}}-\epsilon_{\nu}\right)} \phi_{\mu} \\
& -\sum_{\mu \neq \nu} \frac{\left\langle\phi_{\nu}\left|\delta^{[1]} \hat{H}\right| \phi_{\nu}\right\rangle\left\langle\phi_{\mu}\left|\delta^{[1]} \hat{H}\right| \phi_{\nu}\right\rangle}{\left(\epsilon_{\mu}-\epsilon_{\nu}\right)^{2}} \phi_{\mu} \\
& -\frac{1}{2} \sum_{\mu \neq \nu} \frac{\left|\left\langle\phi_{\mu}\left|\delta^{[1]} \hat{H}\right| \phi_{\nu}\right\rangle\right|^{2}}{\left(\epsilon_{\mu}-\epsilon_{\nu}\right)^{2}} \phi_{\mu} .
\end{aligned}
$$

The real question is whether the perturbation expansion does work for the particle in the trapezoid-well potential of infinite depth in the framework of the bandlimited quantum mechanics. This essentially depends on whether the matrix elements $\left\langle\phi_{\nu}\left|\delta^{[n]} \hat{H}\right| \phi_{\nu^{\prime}}\right\rangle$ do survive the limit $V_{\infty} \rightarrow \infty$ and are of the order $(a / \ell)^{n}$. This will be shown in the next section.

It should be noticed that the formulas in Eqs. (18)(21) are valid for discrete spectrum, but we consider only the low-lying states with energies $\epsilon_{\nu} \ll V_{\infty}$ when the contribution of the continuous spectrum can be neglected and it disappears completely in the limit $V_{\infty} \rightarrow \infty$ that we have taken finally.

\section{GUP EFFECT ON A PARTICLE IN THE CAVITY}

In this section we investigate how GUP affects the lowlying stationary states of the particle in the cavity rep- resented by the trapezoid-well potential of infinite depth in the framework of the perturbation scheme outlined in the previous section, restricting ourselves to the second order of the perturbation expansion in the small parameter $a / \ell$. For this purpose, one needs to evaluate the matrix elements $\left\langle\phi_{\nu}\left|\delta^{[n]} \hat{H}\right| \phi_{\nu^{\prime}}\right\rangle$ for $n=1,2$ of the perturbations in the basis of the unperturbed wavefunctions $\phi_{\nu}$. The determination of the wavefunctions $\phi_{\nu}$ of the bound stationary states in the trapezoid-well potential is an analytically solvable problem in ordinary quantum mechanics given in Appendix A in detail for the case when $V_{\infty}$ is asymptotically large. In order to solve the stationary Schrödinger equation (9) for a particle in the potential $V_{t}(x)$, one has to divide the real axis into the intervals $I_{i}$ with $i=I, I I, I I I, I V, V$ given as

$$
\begin{aligned}
& I_{I}=(-\infty,-b-R], \quad I_{I I}=[-b-R,-b+R], \\
& I_{I I I}=[-b+R, b-R], \\
& I_{I V}=[b-R, b+R], \quad I_{V}=[b+R, \infty)
\end{aligned}
$$

with boundaries at the points of discontinuities of the first derivative of the potential $V_{t}(x)$ and $b=\ell / 2$. In Appendix A we have followed the usual procedure of making an ansatz for the pieces of the wavefunction in the various intervals, then matching the neighbouring pieces by the conditions of continuity of the wavefunction and its first derivative, and finally normalizing the wavefunction. After a straightforward but lengthy calculation the following expression is found for the wavefunction $\phi_{\nu}$ for asymptotically large values of the depth $V_{\infty}$ of the potential well,

$$
\begin{aligned}
\phi_{\nu I}(x) & \sim \frac{1}{2} \pi^{-1 / 2}(\gamma 2 R)^{-1 / 4} e^{-\zeta} B e^{\kappa_{\nu}(x-(-b-R))}, \\
\phi_{\nu I I}(x) & \sim B \operatorname{Ai}\left(\gamma\left(-x-(b-R)-r_{\nu}\right)\right), \\
\phi_{\nu I I I}(x) & \sim \frac{(-i)^{\nu}}{2 \sqrt{b-R}}\left[e^{i k_{\nu} x}+(-1)^{\nu} e^{-i k_{\nu} x}\right],
\end{aligned}
$$

where $\phi_{\nu i}(x)$ denote the pieces of the wavefunction in the intervals $I_{i}$ and the other pieces are given by the parity relations $\phi_{\nu}(x)=(-1)^{\nu} \phi_{\nu}(-x)$ due to the symmetry of the potential $V_{t}(-x)=V_{t}(x)$. Here $\operatorname{Ai}(x)$ stands for the Airy function falling down asymptotically for $x \rightarrow \infty$, and the other notations are

$$
B \sim \frac{k_{\nu}^{\infty}}{\gamma c_{2} \sqrt{b-R}},
$$

$k_{\nu}^{\infty}=\pi(\nu+1) /(\ell-2 R), \quad k_{\nu}=\sqrt{2 m \epsilon_{\nu}} / \hbar, \quad \kappa_{\nu}=$ $\sqrt{2 m\left(V_{\infty}-\epsilon_{\nu}\right)} / \hbar, \quad \gamma=\left(m V_{\infty}\right)^{1 / 3}\left(\hbar^{2} R\right)^{-1 / 3}, \quad r_{\nu}=$ $2 R \epsilon_{\nu} / V_{\infty}, R_{\nu}=2 R-r_{\nu}$, and $\zeta=(2 / 3)(\gamma 2 R)^{3 / 2}$, and $c_{2}=3^{-1 / 3} / \Gamma(1 / 3) \approx 0.259$ as given in Appendix A. It is easy to notice that the wavefunctions (23) are real both for $\nu$ even and odd.

It is worthwhile mentioning that in the limit $V_{\infty} \rightarrow$ $\infty$, i.e., in the limit of the infinite potential depth the pieces $\phi_{\nu I}(x)$ and $\phi_{\nu V}(x)$ of the wavefunctions are suppressed exponentially, due to their constant coefficients, the pieces $\phi_{\nu I I}(x)$ and $\phi_{\nu I V}(x)$ are suppressed due to 
their constant coefficients by the factor $1 / \gamma \sim V_{\infty}^{-1 / 3}$ and only the pieces $\phi_{\nu I I I}(x)$ exhibit coefficients of the order $V_{\infty}^{0}=1$. As to the pieces $\phi_{\nu I I}(x)$ and $\phi_{\nu I V}(x)$, one has to be aware of the fact that those are the wavefunctions in the rather short intervals $I_{I I}$ and $I_{I V}$, so that the arguments of the Airy functions are close to $\gamma\left(R-r_{\nu}\right)$ that takes asymptotically large positive values for $V_{\infty} \rightarrow \infty$. Consequently, the wavefunctions are suppressed exponentially by the factor $e^{-\zeta}$ in the intervals $I_{I I}$ and $I_{I V}$ rather than by the negative power $V_{\infty}^{-1 / 3}$ of $V_{\infty}$. Therefore, the stationary wavefunctions in the infinitely deep trapezoid-well potential contract to the interval $I_{I I I}$, where the potential is vanishing, and satisfy Dirichlet's boundary condition at $x= \pm(b-R)$. In that limit they become identical to the wavefunctions of the stationary states in a box (in a square-well potential of infinite depth) of size $\ell-2 R$.

Let us turn now to the determination of the matrix elements $\left\langle\phi_{\nu}\left|\delta^{[n]} \hat{H}\right| \phi_{\nu^{\prime}}\right\rangle$ for $n=1,2$. Before going into any details, it is in order to make a remark on our expectations how those should behave in the limit $V_{\infty} \rightarrow \infty$. The wavefunction of any state $\nu$ goes essentially over into the wavefunction of the state with the same quantum number $\nu$ in the box (in the square-well potential of infinite depth) of the size $\ell-2 R$. Therefore, we expect that the matrix elements will contain the contributions obtained for the square-well potential, but with the change of the size of the box from $\ell$ to $\ell-2 R$. There may occur however modifications because the size $2 R$ of the intervals $I_{I I}$ and $I_{I V}$ is kept unaltered during the limiting process $V_{\infty} \rightarrow \infty$. These intervals, where the boundaries of the cavity are smeared out have no analogues in the case of the square-well potential, and may alter the matrix elements. Therefore, one cannot say a priori how the matrix elements look like, and their evaluation is then unavoidable.

The matrix elements of any Hermitian symmetric kernel $K(x, y)=K^{*}(y, x)$ can be rewritten as the sum

$$
\int_{-\infty}^{\infty} d x \int_{-\infty}^{\infty} d y \phi_{\nu^{\prime}}^{*}(x) K(x, y) \phi_{\nu}(y)=\sum_{i, j=I}^{V} K_{\nu^{\prime} \nu}^{i j}
$$

with

$$
K_{\nu^{\prime} \nu}^{i j}=\int_{I_{i}} d x \int_{I_{j}} d y \phi_{\nu^{\prime} i}^{*}(x) K(x, y) \phi_{\nu j}(y) .
$$

These integrals are not independent. The kernels we have to do with are real symmetric ones, $K(x, y)=K(y, x)$ and the pieces of the wavefunctions are also real, that implies the relation $K_{\nu^{\prime} \nu}^{i j}=K_{\nu \nu^{\prime}}^{j i}$. Therefore we have to determine the integrals $(26)$ only for $(i j)=(I, I),(I, I I)$, $(I, I I I),(I I, I I),(I I, I I I),(I I I, I I I)$ for an arbitrary choice $\left(\nu^{\prime} \nu\right)$ of the pair of stationary states.

Let us consider the behaviour of the integrals (26) in the limit $V_{\infty} \rightarrow \infty$ for the various kernels in Eq. (11). After factorizing out the coefficients $A, B, D$ of the functions $\phi_{\nu I}(x)$ and $\phi_{\nu I I I}(x)$, their remaining terms exhibit $V_{\infty}$-dependence only through $k_{\nu}$ that takes the finite limit $k_{\nu}^{\infty}$. Furthermore, the kernels $h_{t}(x, y)$ and $t(x, y)$ are independent of the parameter $V_{\infty}$. Therefore the integrals $\left(h_{t}\right)_{\nu^{\prime} \nu}^{I, I},\left(h_{t}\right)_{\nu^{\prime} \nu}^{I, I I I}, t_{\nu^{\prime} \nu}^{I, I}$, and $t_{\nu^{\prime} \nu}^{I, I I I}$ vanish in the limit $V_{\infty} \rightarrow \infty$. In the integrals $v_{\nu^{\prime} \nu}^{I, I}$ and $v_{\nu^{\prime} \nu}^{I, I I I}$ the kernel $v(x, y)$ contains an additional factor of $V_{\infty}$ in both or one of its terms, respectively. This, however, cannot compensate the exponential suppression caused by the factor $e^{-\zeta}$ in $\phi_{\nu I}(x)$, so that these integrals vanish in the limit $V_{\infty} \rightarrow \infty$ too. There is another trivial integral among the $K_{\nu^{\prime} \nu}^{i j}$ 's. Namely, $v_{\nu^{\prime} \nu}^{I I, I I I}=0$ vanishes identically, because the potential $V_{t}(x)$ vanishes in the interval $I_{I I I}$. It should also be noted that the integrals where either one or both of the integration variables are from the interval $I_{I I}$ contain the Airy-function in their integrands with a factor $\gamma \sim V_{\infty}^{1 / 3}$ in its argument, so that the integral should be taken first for finite but large $V_{\infty}$ in order to determine the limit $V_{\infty} \rightarrow \infty$. The integrals $K_{\nu^{\prime} \nu}^{i j}$ not vanishing trivially in the limit $V_{\infty} \rightarrow \infty$ are then $\left(v^{[1]}\right)_{\nu^{\prime} \nu}^{I, I I}$, $\left(v^{[1]}\right)_{\nu^{\prime} \nu}^{I I, I I I},\left(v^{[1]}\right)_{\nu^{\prime} \nu}^{I I, I I},\left(t^{[1]}\right)_{\nu^{\prime} \nu}^{I I, I I I},\left(t^{[1]}\right)_{\nu^{\prime} \nu}^{I, I I},\left(t^{[1]}\right)_{\nu^{\prime} \nu}^{I I, I I I}$, and $\left(t^{[1]}\right)_{\nu^{\prime} \nu}^{I I, I I}$ in the first order and $\left(h_{t}^{[2]}+t^{[2]}\right)_{\nu^{\prime} \nu}^{I I I, I I I}$, $\left(h_{t}^{[2]}+t^{[2]}\right)_{\nu^{\prime} \nu}^{I, I I},\left(h_{t}^{[2]}+t^{[2]}\right)_{\nu^{\prime} \nu}^{I I, I I I}$, and $\left(h_{t}^{[2]}+t^{[2]}\right)_{\nu^{\prime} \nu}^{I I, I I}$ in the second order. The determination of these integrals is given in Appendix $\mathrm{C}$ and can be summarized as follows.

The main conclusion of Appendix $\mathrm{C}$ is that in the limit $V_{\infty} \rightarrow \infty$ all of the matrix elements we need in the perturbation scheme reduce to a single contribution coming from the interval $I_{I I I}$ where the potential $V_{t}$ vanishes,

$$
\begin{aligned}
& \left(v^{[1]}\right)_{\nu^{\prime} \nu} \sim\left(v^{[1]}\right)_{\nu^{\prime} \nu}^{I I I, I I I}, \quad\left(t^{[1]}\right)_{\nu^{\prime} \nu} \sim\left(t^{[1]}\right)_{\nu^{\prime} \nu}^{I I I, I I I}, \\
& \left(h_{t}^{[2]}+t^{[2]}\right)_{\nu^{\prime} \nu} \sim\left(h_{t}^{[2]}+t^{[2]}\right)_{\nu^{\prime} \nu}^{I I I, I I I} .
\end{aligned}
$$

Moreover, the indirect GUP effect coming from the projection of the potential vanishes $\left(v^{[1]}\right)_{\nu^{\prime} \nu} \sim 0$ trivially, because the potential $V_{t}(x)=0$ in the interval $I_{I I I}$, and the other nonvanishing matrix elements responsible for the direct and indirect GUP effects on the kinetic energy operator are diagonal in the state indices,

$$
\begin{aligned}
\left(t^{[1]}\right)_{\nu^{\prime} \nu} & \sim \delta_{\nu^{\prime} \nu} \frac{\hbar^{2}\left(k_{\nu}^{\infty}\right)^{2}}{2 m}\left[\mathcal{I}_{K}(0)-1\right], \\
\left(h_{t}^{[2]}+t^{[2]}\right)_{\nu^{\prime} \nu} & \sim \delta_{\nu^{\prime} \nu} \frac{2\left(k_{\nu}^{\infty} a\right)^{2}}{3} \frac{\hbar^{2}\left(k_{\nu}^{\infty}\right)^{2}}{2 m} \mathcal{I}_{K}(0),
\end{aligned}
$$

where the asymptotic expression

$\mathcal{I}_{K}(0) \approx 1-\frac{2}{\pi}\left(\frac{2 a \cos (K(b-R))}{\pi(\ell-2 R)}-\frac{4 a^{2} \sin (K(b-R))}{\pi^{2}(\ell-2 R)^{2}}\right)$

is reliable due to the large cutoff $K=\pi / a$. In the matrix element $\left(h_{t}^{[2]}+t^{[2]}\right)_{\nu^{\prime} \nu}$ we have to keep only the leading order term of $\mathcal{O}\left(a^{2}\right)$ due to the direct GUP effect when restricting ourselves to the second order of the perturbation expansion. The projection of the ordinary kinetic energy operator results in a sum of first and second order terms (when one neglects the higher-order ones in the asymptotic expansion of $\left.\mathcal{I}_{K}(0)\right)$. Therefore, collecting the first and the second order terms appropriately, 
we should write

$$
\begin{aligned}
&\left\langle\phi_{\nu^{\prime}}\left|\delta^{[1]} H\right| \phi_{\nu}\right\rangle \sim-\delta_{\nu^{\prime} \nu} \frac{\hbar^{2}\left(k_{\nu}^{\infty}\right)^{2}}{2 m} \frac{4 a \cos (K(b-R))}{\pi^{2}(\ell-2 R)}, \\
&\left\langle\phi_{\nu^{\prime}}\left|\delta^{[2]} H\right| \phi_{\nu}\right\rangle \approx \delta_{\nu^{\prime} \nu} \frac{\hbar^{2}\left(k_{\nu}^{\infty}\right)^{2}}{2 m}\left(\frac{2\left(k_{\nu}^{\infty} a\right)^{2}}{3}\right. \\
&\left.+\frac{8 a^{2} \sin (K(b-R))}{\pi^{3}(\ell-2 R)^{2}}\right)
\end{aligned}
$$

for $\hbar \alpha=a$. Here the first-order matrix element occurs purely due to the indirect GUP effect, while the first and second terms of the second-order matrix element occured due to the direct and indirect GUP effects, respectively. We see that the matrix elements (30) of the perturbation Hamiltonian survive the limit increasing the depth $V_{\infty}$ of the trapezoid-well potential to infinity. Furthermore, we also see that in that limit the orders of magnitude of the various terms of the matrix elements (30) are clearly given by their either linear or quadratic dependence on the small parameter $a / \ell$. Therefore the indirect GUP effect can be treated as perturbation that justifies our working hypothesis. This feature is, however, specific for the case when the potential well has infinite depth. For finite depth of the trapezoid-well potential there appear nonvanishing contributions to the matrix elements of the perturbation Hamiltonian which have nonanalytic dependence on the length scale $a$ that is introduced by the projection. This casts doubt on the treatment of the indirect GUP effect as perturbation when the depth of the potential well is finite.

Making use of the formulas (18) and (20) of the stationary perturbation expansion we find the corresponding relative energy corrections of the energy levels,

$$
\begin{aligned}
\frac{\delta^{[1]} \epsilon_{\nu}}{\epsilon_{\nu}} & =\frac{\left\langle\phi_{\nu^{\prime}}\left|\delta^{[1]} H\right| \phi_{\nu}\right\rangle}{\epsilon_{\nu}}=-\frac{4 \cos (K(b-R))}{\pi^{2}} \frac{a}{\ell-2 R},(31) \\
\frac{\delta^{[2]} \epsilon_{\nu}}{\epsilon_{\nu}} & =\frac{\left\langle\phi_{\nu^{\prime}}\left|\delta^{[2]} H\right| \phi_{\nu}\right\rangle}{\epsilon_{\nu}} \\
& =\left(\frac{2 \pi^{2}(\nu+1)^{2}}{3}+\frac{8 \sin (K(b-R))}{\pi^{3}}\right)\left(\frac{a}{\ell-2 R}\right)^{2} .
\end{aligned}
$$

In Table I we compare the results obtained now with those found in our previous paper [1] for the square-well potential of infinite depth. For comparison we have used the asymptotic expansion

$$
\begin{aligned}
\mathcal{I}_{K}(\ell / 2)-1 & =\frac{2}{\pi} \operatorname{Si}(K \ell / 2)-1 \\
& \sim-\frac{2}{\pi}\left(\frac{2 a \cos (K \ell / 2)}{\pi \ell}+\frac{4 a^{2} \sin (K \ell / 2)}{\pi^{2} \ell^{2}}\right)
\end{aligned}
$$

Here we made use of the diagonal behaviour of the matrix elements in the state indices. The first order contribution to the relative energy shift, (31) is of the order $\mathcal{O}(a / \ell)$ and occurs purely due to the indirect GUP effect, while the second order one, (32) is of the order $\mathcal{O}\left((a / \ell)^{2}\right)$ and occurs partially due to the direct GUP effect (the first term in the right-hand side of Eq. (32)) and partially due to the indirect one (the second term in the right-hand side of Eq. (32)). The direct GUP effect results in a positive relative energy shift that increases proportionally to $(\nu+1)^{2}$, i.e., proportionally to the energy $\epsilon_{\nu}$ of the state. The indirect GUP effect is identical for all low-lying stationary states (keeping terms up to second order), but depends periodically on the product $K(b-R)=\frac{1}{2}(\pi / a)(\ell-2 R)$ and its sign alternates both in the first and in the second orders. At this point we have to remember that all the approximations made during the derivation of the asymptotic form of the unperturbed wavefunction (23) in Appendix A and during the evaluation and estimation of the matrix elements for infinite potential depth in Appendix $\mathrm{C}$ remain valid even if the parameter $2 R$ is set as small as the magnitude of the minimal length scale, $2 R \sim a$. The latter choice means that the boundaries of the trapezoid-well potential are smeared out in an interval of the size of the minimal length scale. As to the indirect GUP effect, the proper choice of the size of the boundary regions seems however not to be very important, because its periodic behaviour depends rather on the effective size $\ell-2 R$ of the cavity. Were the length of the cavity quantized as argued in the case of the square-well potential of infinite depth in Refs. [47, 71], i.e., would it hold the relation $\ell-2 R=$ integer $\times 2 a$, the indirect GUP effect of the first order would have alternating sign when this length increases with the amount of a single 'quantum' $2 a$. One should also mention that the wavefunctions keep their unperturbed forms in both the first and second orders of the perturbation expansion due to the diagonality of the matrix elements $\left\langle\phi_{\nu^{\prime}}\left|\delta^{[n]} H\right| \phi_{\nu}\right\rangle \sim \delta_{\nu^{\prime} \nu}$ with $n=1,2$ in the limit $V_{\infty} \rightarrow \infty$.

in order to rewrite our previous result on $R_{t}$ in [1]. We see in Table I that both potentials yield essentially the same relative energy shift $R_{h}$ of the order $(a / \ell)^{2}$ due to the direct GUP effect. In the expression of $R_{h}$ there figures the length of the interval in which the potential vanishes, i.e., $\ell-2 R$ and $\ell$ for the trapezoid-well and the square-well potentials, respectively. The most important change is that no indirect GUP effect occurs due to the projection of the potential operator in the case of the trapezoid-well potential. This is the consequence of the 
TABLE I. Comparison of the various GUP effects for the trapezoid-well and the square-well potentials of infinite depth.

\begin{tabular}{|c|c|c|}
\hline \hline Type of effect & Trapezoid-well potential & Square-well potential \\
\hline \hline$R_{h}$ & $\frac{2}{3}(c \pi)^{2}\left(\frac{a}{\ell-2 R}\right)^{2}(\nu+1)^{2}$ & $\frac{2}{3}(c \pi)^{2}\left(\frac{a}{\ell}\right)^{2}(\nu+1)^{2}$ \\
\hline$R_{v}$ & 0 & $\frac{2}{\pi^{2}} \frac{a}{\ell}\left(2+\left[(\nu+1)^{2} \pi^{2}-2\right] \cos (K \ell)\right)$ for even $\left.\nu\right)$ \\
0 for odd $\nu$ \\
\hline$R_{t}$ & $-\frac{4}{\pi^{2}} \frac{a}{\ell-2 R} \cos (K(\ell-2 R) / 2)+\frac{8}{\pi^{3}}\left(\frac{a}{\ell-2 R}\right)^{2} \sin (K(\ell-2 R) / 2)$ & $-\frac{4}{\pi^{2}} \frac{a}{\ell} \cos (K \ell / 2)-\frac{8}{\pi^{3}}\left(\frac{a}{\ell}\right)^{2} \sin (K \ell / 2)$ \\
\hline \hline
\end{tabular}

existence of the intervals $I_{I I}$ and $I_{I V}$ of finite size $2 R$ where the trapezoid-well potential is smeared out. The width of these intervals remain unaltered when the limit $V_{\infty} \rightarrow \infty$ is taken and consequently the limits of the matrix elements of the projected potential operator are not identical for the trapezoid-well and the square-well potentials. It is worthwhile mentioning that the usage of the trapezoid-well potential leads to relative energy shifts $R_{v}=0$ for all low-lying stationary states, independently of their parity, as opposed to the peculiar result for the square-well potential. The indirect GUP effect occurring due to the projection of the kinetic energy operator causes quite similar relative energy shifts $R_{t}$ for both kinds of potentials, containing terms of both first and second orders. The size of the interval in which the potential vanishes figures in the explicit formulas for $R_{t}$ again. The smearing out of the boundaries of the cavity does not removed the oscillatory behaviour of the relative energy shift $R_{t}$.

\section{SUMMARY}

Summarizing, we have reconsidered the "particle in the box' problem in the framework of one-dimensional bandlimited quantum mechanics by (i) using the infinitely deep trapezoid-well potential with smeared out boundaries, and (ii) applying the nondegenerate stationary perturbation expansion to the determination of the lowenergy stationary states by keeping track of the contributions of various orders to the direct and indirect GUP effect. In that manner we confirmed the qualitative result found in [1] in a more reliable framework. Namely, it is shown that the relative energy shift of the low-energy stationary states of the nonrelativistic particle in the infinitely deep trapezoid-well potential show up a contribution of the order $\ell_{P} / \ell$ caused by the indirect GUP effect, while the direct GUP effect on the energy shift is of second order $\left(\ell_{P} / \ell\right)^{2}$, where $\ell_{P}$ and $\ell$ are the Planck length and the length of the cavity, respectively. We argued that perturbative treatment of the indirect GUP effect may not be possible for the potential well of finite depth.

\section{ACKNOWLEDGEMENTS}

S. Nagy acknowledges the financial support from the János Bolyai Programme of the Hungarian Academy of Sciences.
[1] K. Sailer, Z. Peli, and S. Nagy, Phys. Rev. D 87, 084056 (2013) [arXiv:1301.6913[math-ph]].

[2] M. Maggiore, Phys. Rev. D 49, 5182 (1994).

[3] A. Kempf, String/Quantum Gravity motivated Uncertainty Relations and Regularisation in Field Theory, pp. 6 [arXiv:hep-th/9612082].

[4] C. Bambi, Class. Quant. Grav. 25, 105003 (2008) [arXiv:0804.4746[gr-qc]].
[5] R.J. Adler, Am. J. Phys. 78, 925 (2010) [arXiv:1001.1205[gr-qc]].

[6] K. Nozari, P. Pedram, and M. Molkara, Int. J. Theor. Phys. 51, 1268 (1012) [arXiv:1111.2204[gr-qc]].

[7] G.M. Hossain, V. Husain, and S.S. Seahra, Background independent quantization and the uncertainty principle, pp. 6 [arXiv:1003.2207[gr-qc]]. 
[8] S. Hossenfelder, Minimal Length Scale Scenarios for Quantum Gravity, pp. 86 [arXiv:1203.6191[gr-qc]].

[9] I. Pikowski, M.R. Wanner, M. Aspelmeyer, M.S. Kim, and C. Brukner, Nature Physics 8, 393 (2012) [arXiv:1111.1979[quant-ph]].

[10] C.A. Mead, Phys. Rev. 135, B849 (1964)

[11] J. Wheeler, Ann. Phys. 2, 604 (1957); in Relativity, Groups and Topology, edited by B.S. and C.M. DeWitt (Gordon and Breach, New York, 1963); G.W. Gibbons and S.W. Hawking, Phys. Rev. D 15, 2752 (1977); S. W. Hawking, in General Relativity - An Einstein Centenary Survey, edited by S.W. Hawking and W. Israel (Cambridge University Press, Cambridge, England, 1979).

[12] G. Veneziano, Europhys. Lett. 2, 199 (1986); D. Amati, M. Ciafaloni, and G. Veneziano, Phys. Lett. B 197, 81 (1987); Int. J. Mod. Phys. A 3, 1615 (1988); Phys. Lett. B 216, 41 (1989); Nucl. Phys. B 347, 530 (1990); D.J. Gross and P.F. Mende, Phys. Lett. B 197, 129 (1987); Nucl. Phys. B 303, 407 (1988); K. Konishi, G. Paffuti, and P. Provero, Phys. Lett. B 234, 276 (1990); R. Guida, K. Konishi, and P. Provero, Mod. Phys. Lett. A 6, 1487 (1991); M. Kato, Phys. Lett. B 245, 43 (1990); M. Maggiore, Phys. Lett. B 304, 65 (1993).

[13] B.N. Tiwari, On Generalized Uncertainty Principle, pp. 29 [arXiv:0801.3402[hep-th]].

[14] L.N. Chang, D. Minic, N. Okamura, and T. Takeuchi, Phys. Rev. D 65, 125027 (2002)[arXiv:hep-th/0111181].

[15] L.N. Chang, D. Minic, N. Okamura, and T. Takeuchi, Phys. Rev. D 65, 125028 (2002) [arXiv:hep-th/0201017].

[16] S. Benczik, D. Minic, N. Okamura, S. Rayyan, and T. Takeuchi, Phys. Rev. D 66, 026003 (2002) [arXiv:hepth/0204049].

[17] S. Benczik, D. Minic, N. Okamura, S. Rayyan, and T. Takeuchi, Classical Implications of the Minimal Length Uncertainty Relation, pp. 12, [arXiv:hep-th/0209119].

[18] L.N. Chang, Some Consequences of the Hypothesis of Minimal Lengths, pp. 8, [arXiv:hep-th/0405059].

[19] S. Benczik, L.N. Chang, D. Minic, and T. Takeuchi, Phys. Rev. A 72, 012104 (2005) [arXiv:hep-th/0502222].

[20] S. Benczik, Investigations on the minimal-length uncertainty relation, pp. 87, Ph.D. Thesis, Virginia Tech. (2007) UMI-32-49454.

[21] L.N. Chang, Z. Lewis, D. Minic, and T. Takeuchi, Adv. in High Energy Phys. 2011, 493514 (2011) [arXiv:1106.0068 [hep-th]].

[22] Z. Lewis and T. Takeuchi, Phys. Rev. D 84, 105029 (2011) [arXiv:1109.2680].

[23] N. Arkani-Hamed, S. Dimopoulos, and G. Dvali, Phys. Lett. B 429, 263 (1998) [arXiv:hep-ph/9803315]; Phys. Rev. D 59, 086004 (1999) [arXiv:hep-ph/9807344]; I. Antoniadis, N. Arkani-Hamed, S. Dimopoulos, and G. Dvali, Phys. Lett. B 436, 257 (1998) [arXiv:hep-ph/9804398].

[24] A. Kempf, G. Mangano, and R.B. Mann, Phys. Rev. D 52, 1108 (1995) [arXiv:hep-th/9412167].

[25] A. Kempf, J. Phys. A 30, 2093 (1997) [arXiv:hepth/9604045].

[26] P. Pedram, Phys. Rev. D 85, $024016 \quad$ (2012) [arXiv:1112.2327[hep-th]].

[27] A. Kempf, J. Math. Phys. 35, 4483 (1994) [arXiv:hepth/9311147].

[28] H. Hinrichsen and A. Kempf, J. Math. Phys. 37, 2121 (1996) [arXiv:hep-th/9510144].

[29] A. Kempf, J. Math. Phys. 38, 1342 (1997) [arXiv:hepth/9602085].
[30] S. Detournay, C. Gabriel, and Ph. Spindel, Phys. Rev. D 66, 125004 (2002) [arXiv:hep-th/0210128].

[31] J.Y. Bang and M.S. Berger, Phys. Rev. D 74, 125012 (2006).

[32] G.C. Dorsch and J.A. Nogueira, Int. J. Mod. Phys. A 27, 1250113 (2012) [arXiv:1106.2737[hep-th]].

[33] R.V. Mendes, Searching for the deformation-stability fundamental length (or fundamental time), pp. 35 [arXiv:1111.5576[hep-th]]; R.V. Mendes, Phys.Lett. A376, 1823 (2012) [arXiv:1112.3246 [hep-th]].

[34] C.E. Shannon, The mathematical theory of communication, (The University of Illinois Press, Urbana, 1949).

[35] S. Das, E.C. Vagenas, and A. F. Ali, Phys. Lett. B 690, 407 (2010) [arXiv:1005.3368[hep-th]].

[36] A. Kempf, Three Short Distance Structures from Quantum Algebras, pp. 8, Proceedings 6th Coll. Quantum Groups and Integrable Systems, Prague 19-21 June '97 [arXiv:q-alg/9709023].

[37] A. Kempf, Rept. Math. Phys. 43, 171 (1999) [arXiv:hepth/9806013].

[38] A. Kempf, Phys. Rev. Lett. 85, 2873 (2000) [arXiv:hepth/9905114].

[39] A. Kempf and R. Martin, Phys. Rev. Lett. 100, 021304 (2008) [arXiv:0708.0062[gr-qc]].

[40] A. Kempf, Phys. Rev. Lett. 103, 231301 (2009) [arXiv:0908.3061[gr-qc]].

[41] A. Kempf, New. J. Phys. 12, 115001 (2010) [arXiv:1010.4354[gr-qc]].

[42] M. Bojowald and A. Kempf, Phys. Rev. D 86, 085017 (2012) [arXiv:1112.0994[hep-th]].

[43] A. Kempf, Europhys. Lett. 40, 257 (1997) [arXiv:hepth/9706213].

[44] A. Kempf, On the Structure of Space-Time at the Planck Scale Proc. Erice 98, pp. 10 [arXiv:hep-th/9810215].

[45] A. Kempf, On Symmetric Operators in Noncommutative Geometry, pp. 8, Extended Proc. I.S.I. Guccia Workshop 98, Nova 1999 [arXiv:math-ph/9811027].

[46] A. Kempf, Phys. Rev. D 63, 024017 (2001) [arXiv:hepth/9907160].

[47] A.F. Ali, S. Das, and E.C. Vagenas, Phys. Lett. B 678, 497 (2009) [arXiv:0906.5396[hep-th]].

[48] A.F. Ali, S. Das, and E.C. Vagenas, Phys. Rev. D 84, 044013 (2011) [arXiv:1107.3164[hep-th]].

[49] K. Nozari and T. Azizi, Int. J. Quant. Inf. 3, 623 (2005) [arXiv:gr-qc/0504090].

[50] S. Das and E.C. Vagenas, Phys. Rev. Lett. 101, 221301 (2008) [arXiv:0810.5333[hep-th]]; S. Das and E.C. Vagenas, Can. J. Phys. 87, 233 (2009) [arXiv:0901.1768[hepth]]; M.M. Ettefaghi and S.M. Fazeli, Phys. Rev. Lett. 104, 119001 (2010).

[51] T.K. Jana and P. Roy, SIGMA 5, 083 (2009) [arXiv:0908.1755[quant-ph]].

[52] B. Mirza and M. Zarei, Phys. Rev. D 79, 125007 (2009) [arXiv:0901.1930[hep-th]].

[53] K. Nozari and P. Pedram, Europhys. Lett. 92, 50013 (2010) [arXiv:1011.5673[hep/th]].

[54] A.F. Ali, S. Das, and E.C. Vagenas, The Generalized Uncertainty Principle and Quantum Gravity Phenomenology, pp. 3 [arXiv:1001.2642[math.AG]].

[55] P. Pedram, Europhys. Lett. 89, 50008 (2010) [arXiv:1003.2769[hep-th]].

[56] M. Sprenger, P. Nicolini and M. Bleicher, Class. Quant. Grav. 28, 235019 (2011) [arXiv:1011.5225[hep-ph]]. 
[57] S. Das and R.B. Mann, Phys.Lett. B 704, 596 (2011) [arXiv:1109.3258[hep-th]].

[58] P. Pedram, Phys. Lett. B 714, 317 (2012) [arXiv:1110.2999[hep-th]].

[59] P. Pedram, Int. J. Mod. Phys. D 22, 1350004 (2013) [arXiv:1204.1524[hep-th]].

[60] P. Valtancoli, Mod. Phys. Lett. A 27, 1250107 (2012) [arXiv:1205.3546[hep-th]].

[61] J. Tao, P. Wang, and H. Yang, Homogeneous Field and WKB Approximation In Deformed Quantum Mechanics with Minimal Length, pp. 24, CTP-SCU/2012002 Report [arXiv:1211.5650[hep-th]].

[62] K. Nozari and T. Azizi, Gen. Rel. Grav. 38, 735 (2006) [arXiv:quant-ph/0507018].

[63] P. Pedram, Int. J. Mod. Phys. D 19, 2003 (2010) [arXiv:1103.3805[hep-th]].

[64] P. Pedram, Eur. Phys. J. C73, 2609 (2013) [arXiv:1309.7284 [hep-th]].

[65] A. Boukhellout and D. Bouaziz, Mod. Phys. Lett. A 29, 1450143 (2014) [arXiv:1312.0572 [quant-ph]].

[66] D. Bouaziz and A. Boukhellout, Kratzer's molecular potential in the presence of a minimal length, $17 \mathrm{pp}$. [arXiv:1312.2619 [quant-ph]].

[67] C-L. Ching and R.R. Parwani, Mod. Phys. Lett. A28, $1350061(2013)$

[68] G. Blado, V. Meyers, and C. Owens, Quantum Wells and the Generalized Uncertainty Principle, pp. 15 [arXiv:1312.4876 [physics.chem-ph]].

[69] P.Pedram, Adv. High Energy Phys. 2013, 853696 (2013) [arXiv:1311.7243 [hep-th].

[70] J. Louko, Near-Dirichlet quantum dynamics for a p3corrected particle on an interval, 19 pp. [arXiv:1404.5256 [hep-th]].

[71] V. Balasubramanian, S. Das, and E.C. Vagenas, Generalized Uncertainty Principle and Self-Adjoint Operators, pp. 22 [arXiv:1404.3962 [hep-th]].

[72] M. Abramowitz and I.A. Segun, Handbook of mathematical functions (National Bureau of Standards, Washington, 1972).

\section{Appendix A: Stationary states of a particle in a trapezoid-well potential in ordinary quantum mechanics}

Here we present the solution of the stationary Schrödinger equation (9) with the unperturbed Hamiltonian (8) for the particle in the trapezoid-well potential $V_{t}(x)$ given in Eq. (2) for bound states when the energy of the particle is much less than the depth $V_{\infty}$ of the potential well. In the various intervals $I_{i}$ with $i=I, I I, \ldots, V$ given in (22) the Schrödinger equation can be satisfied by the ansatz

$$
\begin{aligned}
\phi_{\nu I}(x) & =A e^{\kappa_{\nu} x}, \\
\phi_{\nu I I}(x) & =B \operatorname{Ai}\left(f_{I I}(x)\right)+C \operatorname{Bi}\left(f_{I I}(x)\right), \\
\phi_{\nu I I}(x) & =D e^{i k_{\nu} x}+E e^{-i k_{\nu} x}, \\
\phi_{\nu I V}(x) & =F \operatorname{Ai}\left(f_{I V}(x)\right)+G \operatorname{Bi}\left(f_{I V}(x)\right), \\
\phi_{\nu V}(x) & =H e^{-\kappa_{\nu} x}
\end{aligned}
$$

where the coefficients denoted by capital letters from $A$ to $H$ are yet unknown and

$$
k_{\nu}=\sqrt{2 m \epsilon_{\nu}} / \hbar, \quad \kappa_{\nu}=\sqrt{2 m\left(V_{\infty}-\epsilon_{\nu}\right)} / \hbar .
$$

The ansatz for $\phi_{\nu I}(x)$ and $\phi_{\nu V}(x)$ is chosen in such a way that the wavefunction $\phi_{\nu}(x)$ were normalizable, i.e., the exponential functions falling down for $|x| \rightarrow \infty$ were chosen. In the intervals $I_{I I}$ and $I_{I V}$, where the potential is linearly falling and rising, respectively, the Schrödinger equation can be recasted into the form of the differential equation for the Airy functions. Multiplying the Schrödinger equation in the interval $I_{I I}$ by $2 \mathrm{~m} / \hbar^{2}$, one rewrites the equation as

$$
\partial_{x}^{2} \phi_{\nu I I}-\gamma^{3}(-x+\beta) \phi_{\nu I I}=0,
$$

with $\beta=-b+R-r_{\nu}, b=\ell / 2, r_{\nu}=2 R \epsilon_{\nu} / V_{\infty}$, and $\gamma=\left(m V_{\infty}\right)^{1 / 3}\left(\hbar^{2} R\right)^{-1 / 3}$. Then one transforms the independent variable $x$ to $\xi=\gamma(-x+\beta)$ and obtains for the function $\tilde{\phi}_{\nu I I}(\xi(x)) \equiv \phi_{\nu I I}(x)$ the equation

$$
\partial_{\xi}^{2} \tilde{\phi}_{\nu I I}(\xi)-\xi \tilde{\phi}_{\nu I I}(\xi)=0
$$

with the independent solutions $\operatorname{Ai}(\xi)$ and $\operatorname{Bi}(\xi)$, which implies that

$$
f_{I I}(x)=\xi(x)=\gamma(-x+\beta) .
$$

In the interval $I_{I V}$ we get similar expressions for the transformation $x \rightarrow \xi=\gamma(x+\beta)$ and find

$$
f_{I V}(x)=\gamma(x+\beta)=f_{I I}(-x) .
$$

Since the potential is symmetric $V(x)=V(-x)$, the wavefunctions have either positive or negative parities, namely,,,$+-+-\ldots$ in the increasing order of their energies starting from the ground state. When the integer $\nu=0,1,2, \ldots$ enumerates the states with increasing energy, the parity is given by $(-1)^{\nu}$, i.e., $\phi_{\nu}(x)=$ $(-1)^{\nu} \phi_{\nu}(-x)$. This implies the following restrictions on the coefficients

$$
\begin{array}{ll}
H=(-1)^{\nu} A, & F=(-1)^{\nu} B, \\
G=(-1)^{\nu} C, & E=(-1)^{\nu} D .
\end{array}
$$

The remaining 4 independent coefficients have to be determined from the boundary conditions ensuring the continuity of the wavefunction and that of its first derivative,

$$
\begin{aligned}
& A e^{-\kappa(b+R)}=B \operatorname{Ai}\left(f_{I I}(-b-R)\right) \\
& \quad+C \operatorname{Bi}\left(f_{I I}(-b-R)\right), \\
& A \kappa e^{-\kappa(b+R)}=-\gamma\left[B \operatorname{Ai}^{\prime}\left(f_{I I}(-b-R)\right)\right. \\
& \left.\quad+C \operatorname{Bi}^{\prime}\left(f_{I I}(-b-R)\right)\right], \\
& B \operatorname{Ai}\left(f_{I I}(-b+R)\right)+C \operatorname{Bi}\left(f_{I I}(-b+R)\right) \\
& \quad=D\left[e^{i k_{\nu}(-b+R)}+(-1)^{\nu} e^{-i k_{\nu}(-b+R)}\right], \\
& -\gamma\left[B \mathrm{Ai}^{\prime}\left(f_{I I}(-b+R)\right)+C \mathrm{Bi}^{\prime}\left(f_{I I}(-b+R)\right)\right] \\
& \quad=i k_{\nu} D\left[e^{i k_{\nu}(-b+R)}-(-1)^{\nu} e^{-i k_{\nu}(-b+R)}\right],
\end{aligned}
$$


where the prime stands for the differentiation with respect to the argument of the Airy functions, and from the normalization condition

$$
1=\sum_{i=I}^{V} \int_{I_{i}}\left|\phi_{i}\right|^{2} d x .
$$

Because of the relations

$$
\begin{aligned}
& f_{I I}(-b-R)=\gamma R_{\nu} \sim V_{\infty}^{1 / 3}, \\
& f_{I I}(-b+R)=-\gamma r_{\nu} \sim-V_{\infty}^{-2 / 3}
\end{aligned}
$$

with $R_{\nu}=2 R-r_{\nu}>0$ and $r_{\nu}=2 R \epsilon_{\nu} / V_{\infty}>0$, the arguments $f_{I I}(-b-R)$ and $f_{I I}(-b+R)$ take asymptotically large positive and small negative values, respectively, for $V_{\infty}$ tending to infinity, for any $R \geq a$. Therefore we can use the asymptotic formulas $10.4 .59,10.4 .61,10.4 .63$, 10.4.66 in Ref. [72],

$$
\begin{array}{rlrl}
\operatorname{Ai}(z) & \sim \frac{1}{2} \pi^{-1 / 2} z^{-1 / 4} e^{-\zeta}(1+\mathcal{O}(1 / \zeta)), & |\arg z|<\pi \\
\operatorname{Ai}^{\prime}(z) \sim-\frac{1}{2} \pi^{-1 / 2} z^{+1 / 4} e^{-\zeta}(1+\mathcal{O}(1 / \zeta)), & |\arg z|<\pi \\
\operatorname{Bi}(z) \sim \pi^{-1 / 2} z^{-1 / 4} e^{+\zeta}(1+\mathcal{O}(1 / \zeta)), & |\arg z|<\pi / 3 \\
\operatorname{Bi}^{\prime}(z) \sim \pi^{-1 / 2} z^{+1 / 4} e^{+\zeta}(1+\mathcal{O}(1 / \zeta)), & |\arg z|<\pi / 3
\end{array}
$$

with $z=\gamma R_{\nu}$ and $\zeta=\zeta_{\nu}=\frac{2}{3}\left(\gamma R_{\nu}\right)^{3 / 2}$ to obtain the asymptotic expressions

$$
\begin{aligned}
\operatorname{Ai}\left(f_{I I}(-b-R)\right) & =\operatorname{Ai}\left(\gamma R_{\nu}\right) \\
& \sim \frac{1}{2} \pi^{-1 / 2}\left(\gamma R_{\nu}\right)^{-1 / 4} e^{-\zeta_{\nu}} \\
\operatorname{Bi}\left(f_{I I}(-b-R)\right) & =\operatorname{Bi}\left(\gamma R_{\nu}\right) \\
& \sim \pi^{-1 / 2}\left(\gamma R_{\nu}\right)^{-1 / 4} e^{+\zeta_{\nu}} \\
\operatorname{Ai}^{\prime}\left(f_{I I}(-b-R)\right) & =\operatorname{Ai}^{\prime}\left(\gamma R_{\nu}\right) \\
& \sim-\frac{1}{2} \pi^{-1 / 2}\left(\gamma R_{\nu}\right)^{+1 / 4} e^{-\zeta_{\nu}} \\
\operatorname{Bi}^{\prime}\left(f_{I I}(-b-R)\right) & =\operatorname{Bi}^{\prime}\left(\gamma R_{\nu}\right) \\
& \sim \pi^{-1 / 2}\left(\gamma R_{\nu}\right)^{+1 / 4} e^{+\zeta_{\nu}}
\end{aligned}
$$

for asymptotically large values of $V_{\infty}$ and for any $R \geq a$. Furthermore, the expansions 10.4.2-10.4.5 in Ref. [72],

$$
\begin{aligned}
& \operatorname{Ai}(z)=c_{1} f(z)-c_{2} g(z), \\
& \operatorname{Bi}(z)=\sqrt{3}\left[c_{1} f(z)+c_{2} g(z)\right],
\end{aligned}
$$

with $c_{1}=3^{-2 / 3} / \Gamma(2 / 3)=0.355 \ldots, c_{2}=3^{-1 / 3} / \Gamma(1 / 3)=$ $0.2588 \ldots$ and

$$
\begin{aligned}
& f(z)=1+\frac{z^{3}}{3 !}+\mathcal{O}\left(z^{6}\right) \\
& g(z)=z+\frac{2}{4 !} z^{4}+\mathcal{O}\left(z^{7}\right)
\end{aligned}
$$

with $z=-\gamma r_{\nu},|z| \ll 1$ provide the behaviour of the Airy functions and their first derivatives in the neighbourhood of $z=0$, so that one finds

$$
\begin{aligned}
\operatorname{Ai}\left(f_{I I}(-b+R)\right) & =\operatorname{Ai}\left(-\gamma r_{\nu}\right) \approx c_{1}+c_{2} \gamma r_{\nu}, \\
\operatorname{Bi}\left(f_{I I}(-b+R)\right) & =\operatorname{Bi}\left(-\gamma r_{\nu}\right) \approx \sqrt{3}\left(c_{1}-c_{2} \gamma r_{\nu}\right), \\
\operatorname{Ai}^{\prime}\left(f_{I I}(-b+R)\right) & =\operatorname{Ai}^{\prime}\left(-\gamma r_{\nu}\right) \approx-c_{2}+\frac{c_{1}}{2}\left(\gamma r_{\nu}\right)^{2}, \\
\operatorname{Bi}^{\prime}\left(f_{I I}(-b+R)\right) & =\operatorname{Bi}^{\prime}\left(-\gamma r_{\nu}\right) \approx \sqrt{3}\left(c_{2}+\frac{c_{1}}{2}\left(\gamma r_{\nu}\right)^{2}\right) .
\end{aligned}
$$

Making use of the expressions in Eqs. (A15) and (A18), we can rewrite the boundary conditions in Eqs. (A8)(A11) as

$$
\begin{aligned}
& A e^{-\kappa_{\nu}(b+R)}=\frac{1}{2} \pi^{-1 / 2}\left(\gamma R_{\nu}\right)^{-1 / 4}\left(B e^{-\zeta_{\nu}}+2 C e^{+\zeta_{\nu}}\right), \\
& A \kappa_{\nu} e^{-\kappa_{\nu}(b+R)} \frac{1}{2} \pi^{-1 / 2}\left(\gamma R_{\nu}\right)^{+1 / 4} \gamma\left(B e^{-\zeta_{\nu}}-2 C e^{+\zeta_{\nu}}\right), \\
& B\left(c_{1}+c_{2} \gamma r_{\nu}\right)+C \sqrt{3}\left(c_{1}-c_{2} \gamma r_{\nu}\right) \\
& \quad=D\left[e^{i k_{\nu}(-b+R)}+(-1)^{\nu} e^{-i k_{\nu}(-2 L a+R)}\right], \\
& -\gamma\left[B\left(-c_{2}+\frac{c_{1}}{2}\left(\gamma r_{\nu}\right)^{2}\right)+C \sqrt{3}\left(c_{2}+\frac{c_{1}}{2}\left(\gamma r_{\nu}\right)^{2}\right)\right] \\
& \quad=i k_{\nu} D\left[e^{i k_{\nu}(-b+R)}-(-1)^{\nu} e^{-i k_{\nu}(-b+R)}\right] .
\end{aligned}
$$

Dividing the appropriate sides of Eqs. (A19) and (A20) and those of Eqs. (A21) and (A22), we obtain a set of homogeneous linear equations for the determination of the coefficients $B$ and $C$,

$$
\begin{aligned}
& \rho_{\nu}=\frac{B e^{-\zeta_{\nu}}-2 C e^{\zeta_{\nu}}}{B e^{-\zeta_{\nu}}+2 C e^{\zeta_{\nu}}} \\
& \Xi_{\nu}=\gamma \frac{B\left[c_{2}-\frac{c_{1}}{2}\left(\gamma r_{\nu}\right)^{2}\right]-\sqrt{3} C\left[c_{1}+\frac{c_{1}}{2}\left(\gamma r_{\nu}\right)^{2}\right]}{B\left(c_{1}+c_{2} \gamma r_{\nu}\right)+\sqrt{3} C\left(c_{1}-c_{2} \gamma r_{\nu}\right)}
\end{aligned}
$$

where $\rho_{\nu}$ and $\Xi_{\nu}$ on the left-hand sides are given as

$$
\begin{aligned}
\rho_{\nu} & =\frac{\kappa_{\nu}}{\gamma^{3 / 2} R_{\nu}^{1 / 2}} \\
& =\frac{\kappa}{\gamma^{3 / 2}(2 R)^{1 / 2}}\left(1-\frac{\xi_{\nu}}{2}-\frac{\xi_{\nu}^{2}}{8}\right)\left(1+\frac{\xi_{\nu}}{2}+\frac{3 \xi_{\nu}^{2}}{8}\right) \\
& =1+\mathcal{O}\left(\xi_{\nu}^{3}\right)
\end{aligned}
$$

with $0<\xi_{\nu}=\frac{r_{\nu}}{2 R} \ll 1, \kappa=\sqrt{2 m V_{\infty}} / \hbar$ and

$$
\begin{aligned}
\Xi_{\nu} & =i k_{\nu} \frac{e^{i k_{\nu}(-b+R)}-(-1)^{\nu} e^{-i k_{\nu}(-b+R)}}{e^{i k_{\nu}(-b+R)}+(-1)^{\nu} e^{-i k_{\nu}(-b+R)}} \\
& =k_{\nu}\left(\begin{array}{cc}
\tan \left(k_{\nu}(b-R)\right) & \nu=\text { even } \\
-\operatorname{cotan}\left(k_{\nu}(b-R)\right) & \nu=\text { odd }
\end{array}\right),
\end{aligned}
$$

respectively. Eq. (A23) with unity on the left-hand side in the limit $V_{\infty} \rightarrow \infty$ (c.f. Eq. (A25)) can only be satisfied for $C=0$. Otherwise -1 were the limiting value of the right-hand side in contradiction with the limiting 
value +1 of the left-hand side. Then Eq. (A24) reduces to

$$
\Xi_{\nu}=\gamma \frac{c_{2}-\frac{c_{1}}{2}\left(\gamma r_{\nu}\right)^{2}}{c_{1}+c_{2} \gamma r_{\nu}} \sim \gamma \frac{c_{2}}{c_{1}}
$$

that goes to $+\infty$ for $V_{\infty} \rightarrow \infty$. This implies, however, via the singularities of the tangent and cotangent functions in the right-hand side of Eq. (A26) that $k_{\nu}$ can be given as $k_{\nu}=k_{\nu}^{\infty}-\delta k_{\nu}$ with

$$
k_{\nu}^{\infty}=\frac{\pi(\nu+1)}{\ell-2 R}
$$

and the yet undetermined $\delta k_{\nu}$ for which the inequalities $0<\delta k_{\nu} \ll k_{\nu}^{\infty}$ hold for asymptotically large values of $V_{\infty}$. Making use of $C=0$ and Eqs. (A28) and (A26), we can recast Eq. (A24) in the form

$\frac{1}{\gamma} \frac{c_{1}+c_{2} \gamma r_{\nu}}{c_{2}-\frac{c_{1}}{2}\left(\gamma r_{\nu}\right)^{2}}=\frac{1}{k_{\nu}}\left(\begin{array}{cc}\operatorname{cotan}\left(k_{\nu}(b-R)\right) & \nu=\text { even } \\ -\tan \left(k_{\nu}(b-R)\right) & \nu=\text { odd }\end{array}\right)$

and expand the left-hand side in powers of $r_{\nu}$ and the right-hand side in powers of $\delta k_{\nu} / k_{\nu}^{\infty}$ (at the zeroth of the trigonometric functions). We keep the terms up to the first order in $\delta k_{\nu} / k_{\nu}^{\infty}$, while in the other expansion we have kept the terms of second order in $r_{\nu}$ because in that manner we keep all terms of the order $a$ and $a^{2}$ until the limit $V_{\infty} \rightarrow \infty$ is taken. Then we find

$$
\frac{\delta k_{\nu}}{k_{\nu}^{\infty}} \approx \frac{1}{\gamma(b-R)}\left(\frac{c_{1}}{c_{2}}+\gamma r_{\nu}+\frac{c_{1}^{2}}{2 c_{2}^{2}}\left(\gamma r_{\nu}\right)^{2}+\mathcal{O}\left(r_{\nu}^{3}\right)\right) .
$$

The first term in the bracket provides the leading order term for asymptotically large $V_{\infty}$ and $\delta k_{\nu} \rightarrow 0$ in the limit $V_{\infty} \rightarrow \infty$.

Now we have $C=0$ and the coefficients $A$ and $D$ can be expressed from Eqs. (A19) and (A21) in terms of $B$,

$$
\begin{aligned}
A e^{-\kappa_{\nu}(b+R)}= & \frac{1}{2} \pi^{-1 / 2}\left(\gamma R_{\nu}\right)^{-1 / 4} e^{-\frac{2}{3}\left(\gamma R_{\nu}\right)^{3 / 2}} B \\
\approx & \frac{1}{2} \pi^{-1 / 2}(\gamma 2 R)^{-1 / 4} e^{-\zeta}\left(1+\frac{\xi_{\nu}}{4}(1+6 \zeta)\right. \\
& \left.+\frac{\xi_{\nu}^{2}}{32}\left(5+36 \zeta^{2}\right)+\mathcal{O}\left(\xi^{3}\right)\right) B, \quad(\mathrm{~A} 31) \\
D= & \frac{c_{1}+c_{2} \gamma r_{\nu}}{e^{i k_{\nu}(-b+R)}+(-1)^{\nu} e^{-i k_{\nu}(-b+R)}} B \\
\approx & \frac{(-i)^{\nu} \gamma}{2 k_{\nu}^{\infty}}\left(c_{2}-\frac{c_{1}}{2}\left(\gamma r_{\nu}\right)^{2}+\mathcal{O}\left(r_{\nu}^{3}\right)\right) B,
\end{aligned}
$$

where the terms up to the quadratic ones in $\xi_{\nu}$ and $r_{\nu}$ have been kept and $\zeta=\frac{2}{3}(\gamma 2 R)^{3 / 2}$.

As to the next, one has to determine $B$ from the normalization condition (A12) that can be recasted into the following form,

$$
\frac{1}{2}|B|^{-2}=\left|A e^{-\kappa_{\nu}(b+R)} / B\right|^{2} \mathcal{I}_{A}+\mathcal{I}_{B}+|D / B|^{2} \mathcal{I}_{D}
$$

with the integrals

$$
\begin{aligned}
\mathcal{I}_{A} & =\int_{-\infty}^{0} d x e^{2 \kappa x}=\frac{1}{2 \kappa_{\nu}} \\
& \sim \frac{1}{2 \kappa}\left(1+\frac{\xi_{\nu}}{2}+\frac{3 \xi_{\nu}^{2}}{8}+\mathcal{O}\left(\xi_{\nu}^{3}\right)\right), \\
\mathcal{I}_{B} & =\int_{-2 R+r_{\nu}}^{r_{\nu}} d x \operatorname{Ai}^{2}(-\gamma x), \\
\mathcal{I}_{D} & =\int_{-b+R}^{0} d x\left|e^{i k_{\nu} x}+(-1)^{\nu} e^{-i k_{\nu} x}\right|^{2} \\
& =\left(\ell-2 R+\frac{(-1)^{\nu}}{k_{\nu}} \sin \left(k_{\nu}(\ell-2 R)\right)\right) \\
& \sim\left(1+\frac{\delta k_{\nu}}{k_{\nu}^{\infty}}\right)(\ell-2 R)
\end{aligned}
$$

for asymptotically large values of $V_{\infty}$. Since $0<r_{\nu} \ll$ $R$ for sufficiently large $V_{\infty}$ and for any $R \geq a$, we can estimate the integral $\mathcal{I}_{B}$ by expanding it in powers of $r_{\nu}$,

$$
\begin{aligned}
\mathcal{I}_{B} \approx & \int_{-2 R}^{0} d x \operatorname{Ai}^{2}(-\gamma x)+r_{\nu}\left[\operatorname{Ai}^{2}(0)-\operatorname{Ai}^{2}(\gamma 2 R)\right] \\
& -\gamma r_{\nu}^{2}\left[\operatorname{Ai}(0) \operatorname{Ai}^{\prime}(0)-\operatorname{Ai}(\gamma 2 R) \operatorname{Ai}^{\prime}(\gamma 2 R)\right] .
\end{aligned}
$$

Here the integral in the right-hand side is bounded in the limit $V_{\infty} \rightarrow \infty$,

$$
\begin{aligned}
\int_{-2 R}^{0} d x \mathrm{Ai}^{2}(-\gamma x) & =\int_{0}^{\gamma 2 R} d x \mathrm{Ai}^{2}(x) \\
& <\int_{0}^{\infty} d x \mathrm{Ai}^{2}(x) \equiv M_{1},
\end{aligned}
$$

where $M_{1}>0$ is a constant independent of $\gamma$. Making use of the expansion of the Airy function $\operatorname{Ai}(z)$ and that of its first derivative at $z=0$ given in Eqs. (A16) as well as their asymptotic expressions in Eqs. (A15) we find then the estimate

$$
\begin{aligned}
\mathcal{I}_{B} \approx & M_{1}+r_{\nu}\left(c_{1}^{2}-\frac{1}{4 \pi}(\gamma 2 R)^{-1 / 2} e^{-2 \zeta}\right) \\
& -\gamma r_{\nu}^{2}\left(-c_{1} c_{2}+\frac{1}{4 \pi} e^{-2 \zeta}\right) .
\end{aligned}
$$

Making use of Eqs. (A31), (A34) for the integrals $\mathcal{I}_{A}$ and $\mathcal{I}_{D}$, and the estimate $(\mathrm{A} 37)$ of the integral $\mathcal{I}_{B}$, we can rewrite Eq. (A33) into the more explicit form

$$
\begin{aligned}
& \frac{1}{2}|B|^{-2} \frac{e^{-2 \zeta}}{8 \pi \kappa \sqrt{\gamma 2 R}}\left[1+\frac{3 \xi_{\nu}}{4}(1+2 \zeta)+\frac{\xi_{\nu}^{2}}{32}\left(21+24 \zeta+36 \zeta^{2}\right)\right] \\
&+M_{1}+r_{\nu}\left(c_{1}^{2}-\frac{e^{-2 \zeta}}{4 \pi \sqrt{\gamma 2 R}}\right)-\gamma r_{\nu}^{2}\left(-c_{1} c_{2}+\frac{e^{-2 \zeta}}{4 \pi}\right) \\
&+\frac{\gamma^{2} c_{2}^{2}}{2\left(k_{\nu}^{\infty}\right)^{2}}\left[2 L a-R+\frac{c_{1}}{\gamma c_{2}}+r_{\nu}\right. \\
&\left.\quad-\frac{c_{1}}{c_{2}}\left(2 L a-R+\frac{c_{1}}{2 \gamma c_{2}}\right)\left(\gamma r_{\nu}\right)^{2}\right] . \quad
\end{aligned}
$$


Here $e^{-\zeta}, \gamma r_{\nu}$ and $\gamma^{2} r_{\nu}$ vanish in the limit $V_{0} \rightarrow \infty$, therefore the only terms surviving that limit yield

$$
\begin{aligned}
|B|^{2} & \sim \frac{1}{2}\left(\frac{\gamma^{2} c_{2}^{2}(b-R)}{2\left(k_{\nu}^{\infty}\right)^{2}}+\frac{\gamma c_{1} c_{2}}{2\left(k_{\nu}^{\infty}\right)^{2}}+M_{1}\right)^{-1} \\
& \sim \frac{\left(k_{\nu}^{\infty}\right)^{2}}{\gamma^{2} c_{2}^{2}(b-R)}+\mathcal{O}\left(1 / \gamma^{3}\right)
\end{aligned}
$$

in the leading order of $1 / \gamma$ tending to zero.

Up to an irrelevant overall phase factor the asymptotic values of the coefficients of the normalized wavefunctions are therefore

$$
B \sim \frac{k_{\nu}^{\infty}}{\gamma c_{2} \sqrt{b-R}}
$$

and from the expressions in (A31),

$$
\begin{aligned}
A e^{-\kappa_{\nu}(b+R)} & \sim \frac{k_{\nu}^{\infty} e^{-\zeta}}{2 c_{2} \gamma \sqrt{\pi(b-R)}(\gamma 2 R)^{1 / 4}}, \\
D & \sim \frac{(-i)^{\nu}}{2 \sqrt{b-R}} .
\end{aligned}
$$

The asymptotic expressions of the pieces of the wavefunction of the stationary state $\nu$ in the trapezoid-well potential with asymptotically large depth are then given by the Eqs. in (23) and by the parity relations $\phi_{\nu}(x)=$ $(-1)^{\nu} \phi_{\nu}(-x)$, when we do not make the expression of the coefficient $B$ explicit.

\section{Appendix B: Reminder on nondegenerate stationary perturbation expansion}

Let us insert the perturbations expansions in Eqs. (12) and (17) into the stationary Schrödinger equation (3) and write down the equalities of the terms on both sides of the equation order by order. The equation for the terms of zeroth order is just the stationary Schrödinger equation (9) of the unperturbed system. In the first and second orders of the perturbation expansion we find

$$
\begin{aligned}
\hat{H}_{0} \delta^{[1]} \psi_{\nu}+\delta^{[1]} \hat{H} \phi_{\nu}=\epsilon_{\nu} \delta^{[1]} \psi_{\nu}+\delta^{[1]} \epsilon_{\nu} \phi_{\nu}, & (\mathrm{B} 1) \\
\hat{H}_{0} \delta^{[2]} \psi_{\nu}+\delta^{[2]} \hat{H} \phi_{\nu}+\delta^{[1]} \hat{H} \delta^{[1]} \psi_{\nu}= & \epsilon_{\nu} \delta^{[2]} \psi_{\nu}+\delta^{[2]} \epsilon_{\nu} \phi_{\nu} \\
& +\delta^{[1]} \epsilon_{\nu} \delta^{[1]} \psi_{\nu}, \quad(\mathrm{B} 2
\end{aligned}
$$

respectively.

Both the first- $(n=1)$ and second-order $(n=2)$ corrections to the energy and the wavefunctions can be obtained by taking the scalar product of both sides of Eqs. (B1) and (B2), respectively with the wavefunction $\phi_{\mu}$ and expanding the perturbative corrections $\delta^{[n]} \psi_{\nu}$ of the wavefunction in terms of the unperturbed wavefunctions $\phi_{\mu}$,

$$
\delta^{[n]} \psi_{\nu}=c_{\nu \nu}^{[n]} \phi_{\nu}+\sum_{\mu \neq \nu} c_{\nu \mu}^{[n]} \phi_{\mu} .
$$

Then the case with $\mu=\nu$ yields the real energy shifts given in Eqs. (18) and (20) (remind that all operators $\delta^{[n]} \hat{H}$ are Hermitian symmetric by construction) and the case with $\mu \neq \nu$ provides the coefficients

$$
c_{\nu \mu}^{[1]} \equiv\left\langle\phi_{\mu} \mid \delta^{[1]} \psi_{\nu}\right\rangle=-\frac{\left\langle\phi_{\mu}\left|\delta^{[1]} \hat{H}\right| \phi_{\nu}\right\rangle}{\epsilon_{\mu}-\epsilon_{\nu}}
$$

and

$$
\begin{aligned}
c_{\nu \mu}^{[2]}= & -\frac{\left\langle\phi_{\mu}\left|\delta^{[2]} H\right| \phi_{\nu}\right\rangle}{\epsilon_{\mu}-\epsilon_{\nu}} \\
& +\sum_{\mu^{\prime} \neq \nu} \frac{\left\langle\phi_{\mu}\left|\delta^{[1]} H\right| \phi_{\mu^{\prime}}\right\rangle\left\langle\phi_{\mu}^{\prime}\left|\delta^{[1]} H\right| \phi_{\nu}\right\rangle}{\left(\epsilon_{\mu}-\epsilon_{\nu}\right)\left(\epsilon_{\mu^{\prime}}-\epsilon_{\nu}\right)} \\
& -\frac{\left\langle\phi_{\nu}\left|\delta^{[1]} H\right| \phi_{\nu}\right\rangle\left\langle\phi_{\mu}\left|\delta^{[1]} H\right| \phi_{\nu}\right\rangle}{\left(\epsilon_{\mu}-\epsilon_{\nu}\right)^{2}} .
\end{aligned}
$$

The coefficients $c_{\nu \nu}^{[1]}$ and $c_{\nu \nu}^{[2]}$ should be determined from the normalization to unity of the first- and second-order improved wavefunctions, respectively, with the appropriate accuracy. Then one finds $c_{\nu \nu}^{[1]}=0$ and

$$
c_{\nu \nu}^{[2]}=-\frac{1}{2} \sum_{\mu \neq \nu} \frac{\left|\left\langle\phi_{\mu}\left|\delta^{[1]} \hat{H}\right| \phi_{\nu}\right\rangle\right|^{2}}{\left(\epsilon_{\mu}-\epsilon_{\nu}\right)^{2}}
$$

and obtains the expressions in Eqs. (19) and (21) finally.

\section{Appendix C: Evaluation of matrix elements}

In this section we outline the evaluation of the integrals $\left(v^{[1]}\right)_{\nu^{\prime} \nu}^{I I, I I I},\left(v^{[1]}\right)_{\nu^{\prime} \nu}^{I I, I},\left(v^{[1]}\right)_{\nu^{\prime} \nu}^{I I, I I},\left(t^{[1]}\right)_{\nu^{\prime} \nu}^{I I I, I I I},\left(t^{[1]}\right)_{\nu^{\prime} \nu}^{I I, I I I}$, $\left(t^{[1]}\right)_{\nu^{\prime} \nu}^{I I, I I},\left(t^{[1]}\right)_{\nu^{\prime} \nu}^{I I, I},\left(h_{t}^{[2]}+t^{[2]}\right)_{\nu^{\prime} \nu}^{I I I, I I I},\left(h_{t}^{[2]}+t^{[2]}\right)_{\nu^{\prime} \nu}^{I I, I I I}$, $\left(h_{t}^{[2]}+t^{[2]}\right)_{\nu^{\prime} \nu}^{I I, I I}$, and $\left(h_{t}^{[2]}+t^{[2]}\right)_{\nu^{\prime} \nu}^{I I, I}$ for finite but asymptotically large values of $V_{\infty}$. These are the integrals contributing to the matrix elements which may become nonvanishing in the limit $V_{\infty} \rightarrow \infty$. Evaluating the integrals $K_{\nu^{\prime} \nu}^{i j}$ according to Eq. (26) one has to keep in mind that (i) the term containing the Dirac delta does not contribute if the intervals $I_{i}$ and $I_{j}$ are different, and (ii) the wavefunctions are real. One obtains the explicit expressions for the integrals $K_{\nu^{\prime} \nu}^{i j}$ for asymptotically large values of the parameter $V_{\infty}$ by inserting the expressions (16), (14), (13), and (15) of the appropriate kernels and the expressions of the appropriate pieces of the wavefunctions given in Eq. (23) into the double integral in Eq. (26). In order to make our formulas more compact let us use the notations

$$
\begin{aligned}
\int_{I} d x f(x) & =\int_{-\infty}^{-b-R} d x f(x), \\
\int_{I I} d x f(x) & =\int_{-b-R}^{-b+R} d x f(x) \\
\int_{I I I} d x f(x) & =\int_{-b+R}^{b-R} d x f(x)
\end{aligned}
$$

with $b=\ell / 2$. 


\section{Determination of $\left(v^{[1]}\right)_{\nu^{\prime} \nu}^{i j}$}

Let us start with the integral $\left(v^{[1]}\right)_{\nu^{\prime} \nu}^{I I, I I I}$ that reduces to

$$
\begin{aligned}
&\left(v^{[1]}\right)_{\nu^{\prime} \nu}^{I I, I I I} \\
&=\int_{I I} d x \int_{I I I} d y \phi_{\nu^{\prime} I I}(x)^{*} \frac{1}{2}\left[V_{t}(x)+V_{t}(y)\right] \\
& \quad \times \Pi(x-y) \phi_{\nu I I I}(y) \\
& \sim \frac{V_{\infty}}{4 R} \frac{k_{\nu^{\prime}}^{\infty}(-i)^{\nu}}{2 \gamma c_{2}(b-R)} \\
& \times \int_{I I} d x \int_{I I I} d y \operatorname{Ai}\left(\gamma\left(-x-(b-R)-r_{\nu^{\prime}}\right)\right) \\
& \times[(R-x-b)+(R-y-b)] \Pi(x-y) \\
& \times\left[e^{i k_{\nu} y}+(-1)^{\nu} e^{-i k_{\nu} y}\right] .
\end{aligned}
$$

Let us make use of the inequality $2 R \ll \ell$ and expand the integral over the variable $x$ as the function of its limits in the small parameter $2 R / \ell$, keeping the leading order term,

$$
\begin{aligned}
& \left(v^{[1]}\right)_{\nu^{\prime} \nu}^{I I, I I I} \\
& \sim \frac{V_{\infty}}{4 R} \frac{k_{\nu^{\prime}}^{\infty}(-i)^{\nu}}{2 \gamma c_{2}(b-R)} 2 R \operatorname{Ai}\left(\gamma\left(R-r_{\nu^{\prime}}\right)\right) \\
& \quad \times \int_{I I I} d y[R+(R-y-2 L a)] \Pi(-2 L a-y) \\
& \quad \times\left[e^{i k_{\nu} y}+(-1)^{\nu} e^{-i k_{\nu} y}\right] .
\end{aligned}
$$

The remaining integral over the variable $y$ has finite limit, while the argument of the Airy function takes asymptotically large values for $V_{\infty} \rightarrow \infty$ and therefore the Airy-function suppresses exponentially the integral $\left(v^{[1]}\right)_{\nu^{\prime} \nu}^{I I I I I} \rightarrow 0$ in that limit.

Replacing the function $\phi_{\nu I I I}(y)$ by $\phi_{\nu I}(y)$ and changing the integral $\int_{I I I} d y$ to $\int_{I} d y$ in Eq. (C2), we find in a similar manner that $\left(v^{[1]}\right)_{\nu^{\prime} \nu}^{I I, I} \rightarrow 0$ in the limit $V_{\infty} \rightarrow \infty$.

The integral $\left(v^{[1]}\right)_{\nu^{\prime} \nu}^{I I, I I}$ can be rewritten as

$$
\begin{aligned}
\left(v^{[1]}\right)_{\nu^{\prime} \nu}^{I I, I I} & \\
= & \frac{V_{\infty}}{4 R} \frac{k_{\nu^{\prime}}^{\infty} k_{\nu}^{\infty}}{\gamma^{2} c_{2}^{2}(b-R)} \\
& \times \int_{I I} d x \int_{I I} d y \operatorname{Ai}\left(\gamma\left(-x-b+R-r_{\nu^{\prime}}\right)\right) \\
& \times[-\ell+2 R-x-y][\Pi(x-y)-\delta(x-y)] \\
& \times \operatorname{Ai}\left(\gamma\left(-y-(b-R)-r_{\nu}\right)\right) .
\end{aligned}
$$

Let us first expand the integral over $y$ as the functions of its limits in the small parameter $2 R / \ell$, keeping the leading order terms, and then do the same for the $x$ integral. Then one finds

$$
\begin{aligned}
\left(v^{[1]}\right)_{\nu^{\prime} \nu}^{I I, I I} \sim & \frac{V_{\infty}}{4 R} \frac{k_{\nu^{\prime}}^{\infty} k_{\nu}^{\infty}}{\gamma^{2} c_{2}^{2}(b-R)}(2 R) \operatorname{Ai}\left(\gamma\left(R-r_{\nu}\right)\right) \\
& \times\left[(2 R) \operatorname{Ai}\left(\gamma\left(R-r_{\nu^{\prime}}\right)\right)(2 R) \Pi(0)\right. \\
& \left.-2 R \operatorname{Ai}\left(\gamma\left(R-r_{\nu^{\prime}}\right)\right)\right]
\end{aligned}
$$

Because of the Airy functions with arguments tending to plus infinity this integral is exponentially suppressed in the limit $V_{\infty} \rightarrow \infty$.

\section{Determination of $\left(t^{[1]}\right)_{\nu^{\prime} \nu}^{i j}$ and $\left(h_{t}^{[2]}+t^{[2]}\right)_{\nu^{\prime} \nu}^{i j}$}

Let us evaluate now the integrals $K_{\nu^{\prime} \nu}^{i j}$ connected with the projection of the kinetic energy operator. Let us start with the integral $\left(t^{[1]}\right)_{\nu^{\prime} \nu}^{I I I, I I I}$ that can be rewritten as the sum of two integrals, $\left(t^{[1]}\right)_{\nu^{\prime} \nu}^{I I I, I I I}=\left(t_{\Pi}^{[1]}\right)_{\nu^{\prime} \nu}^{I I I, I I I}+$ $\left(t_{D}^{[1]}\right)_{\nu^{\prime} \nu}^{I I I, I I I}$ with the terms containing the projector and the Dirac-delta,

$$
\begin{aligned}
\left(t_{\Pi}^{[1]}\right)_{\nu^{\prime} \nu}^{I I I, I I I} & \\
= & -\frac{\hbar^{2}}{2 m} \frac{i^{\nu^{\prime}-\nu}}{4(b-R)} \int_{I I I} d x \int_{I I I} d y\left[e^{-i k_{\nu^{\prime}} x}\right. \\
& \left.+(-1)^{\nu^{\prime}} e^{i k_{\nu^{\prime}} x}\right] \partial_{x}^{2} \Pi(x-y)\left[e^{i k_{\nu} y}+(-1)^{\nu} e^{-i k_{\nu} y}\right],
\end{aligned}
$$

and

$$
\begin{aligned}
\left(t_{D}^{[1]}\right)_{\nu^{\prime} \nu}^{I I I, I I I} & \\
= & -\frac{\hbar^{2} k_{\nu}^{2}}{2 m} \frac{i^{\nu^{\prime}-\nu}}{4(b-R)} \int_{I I I} d x\left[e^{-i k_{\nu^{\prime}} x}\right. \\
& \left.+(-1)^{\nu^{\prime}} e^{i k_{\nu^{\prime}} x}\right]\left[e^{i k_{\nu} x}+(-1)^{\nu} e^{-i k_{\nu} x}\right],
\end{aligned}
$$

respectively. The integral in the term $\left(t_{D}^{[1]}\right)_{\nu^{\prime} \nu}^{I I I, I I I}$ can be performed easily and yields

$$
\begin{aligned}
& \left(t_{D}^{[1]}\right)_{\nu^{\prime} \nu}^{I I I, I I I} \\
& =-\frac{\hbar^{2} k_{\nu}^{2}}{2 m} \frac{i^{\nu^{\prime}-\nu}\left[1+(-1)^{\nu^{\prime}+\nu}\right]}{2(b-R)}\left(\frac{\sin \left(\left(k_{\nu^{\prime}}-k_{\nu}\right)(b-R)\right)}{k_{\nu^{\prime}}-k_{\nu}}\right. \\
& \left.\quad+(-1)^{\nu} \frac{\sin \left(\left(k_{\nu^{\prime}}+k_{\nu}\right)(b-R)\right)}{k_{\nu^{\prime}}+k_{\nu}}\right) .
\end{aligned}
$$

We can take now the limit $V_{\infty} \rightarrow \infty$ that implies $k_{\nu} \rightarrow$ $k_{\nu}^{\infty}$ and making use of Eq. (A28), one finds

$$
\left(t_{D}^{[1]}\right)_{\nu^{\prime} \nu}^{I I I, I I I}=-\frac{\hbar^{2}\left(k_{\nu}^{\infty}\right)^{2}}{2 m} \delta_{\nu^{\prime} \nu} .
$$

It is straightforward to obtain this result for $\nu^{\prime}=\nu$. For $\nu^{\prime} \neq \nu$ one gets the expression

$$
\begin{aligned}
& \left(t_{D}^{[1]}\right)_{\nu^{\prime} \nu}^{I I I, I I I} \\
& =-\frac{\hbar^{2}\left(k_{\nu}^{\infty}\right)^{2}}{2 m} i^{\nu^{\prime}-\nu}\left[1+(-1)^{\nu^{\prime}+\nu}\right]\left(\frac{\sin \left(\frac{\pi}{2}\left(\nu^{\prime}-\nu\right)\right)}{\pi\left(\nu^{\prime}-\nu\right)}\right. \\
& \left.\quad+(-1)^{\nu} \frac{\sin \left(\frac{\pi}{2}\left(\nu^{\prime}+\nu+2\right)\right)}{\pi\left(\nu^{\prime}+\nu+2\right)}\right)
\end{aligned}
$$

that vanishes if both $\nu^{\prime}$ and $\nu$ are even or odd (i.e., $\nu^{\prime}-\nu$ and $\nu^{\prime}+\nu$ are even) due to the vanishing of the values of the sine functions, and it vanishes also when one of the integers $\nu^{\prime}$ and $\nu$ is even and the other odd (i.e., $\nu^{\prime}-\nu$ is odd) due to the factor in the square bracket. Thus 
one finds the expression in Eq. (C9), that equals to the negative of the unperturbed kinetic energy of the particle in the cavity for $\nu^{\prime}=\nu$ and vanishes for $\nu^{\prime} \neq \nu$. Let us note that the expressions obtained in the limit $V_{\infty} \rightarrow \infty$ are independent of the ratio $(\ell-2 R) / a$.

The evaluation of the term $\left(t_{\Pi}^{[1]}\right)_{\nu^{\prime} \nu}^{I I I, I I I}$ is more cumbersome. In order to estimate this integral, we perform its evaluation similarly to that done for $t_{I I, I I}$ in Eq. (E8) in [1]. First we rewrite the integral $\int_{-d}^{d} d y \delta(x-y) e^{i k y}=$ $e^{i k x} \chi_{[-d, d]}(x)$, where $\chi_{[-d, d]}(x)$ is the characteristic function of the interval $x \in[-d, d]$, as the limit

$$
\int_{-d}^{d} d y \delta(x-y) e^{i k y}=e^{i k x} \lim _{\Lambda \rightarrow \infty} \mathcal{I}_{\Lambda}(x)
$$

with

$$
\mathcal{I}_{\Lambda}(x)=\frac{1}{2} \int_{-1}^{1} d s\left[\frac{\sin [s \Lambda(x+d)]}{s \pi}-\frac{\sin [s \Lambda(x-d)]}{s \pi}\right] .
$$

Then we realize that for $x \in[-d, d]$ the integral $\int_{-d}^{d} d y \Pi(x-y) e^{i k y}$ can be rewritten as

$$
\int_{-d}^{d} d y \Pi(x-y) e^{i k y}=e^{i k x} \mathcal{I}_{K}(x)
$$

Below we have to set $d=b-R$. The cutoff $\Lambda$ has been replaced by $K$ and the limit removed. For extremely large values of $K=\pi / a$ the function $\mathcal{I}_{K}(x)$ should be rather smooth, because in the limit $K \rightarrow \infty$ it tends to 1 , a value independent of $x$. Therefore, we can write as a good approximation

$$
\partial_{x}^{2} \int_{-d}^{d} d y \Pi(x-y) e^{i k y} \approx-k^{2} e^{i k x} \mathcal{I}_{K}(x)
$$

for $x \in[-d, d]$, neglecting the terms with the derivatives of $\mathcal{I}_{K}(x)$. Now the first integral term of $\left(t^{[1]}\right)_{\nu^{\prime} \nu}^{I I, I I I}$ can be estimated as

$$
\begin{aligned}
\left(t_{\Pi}^{[1]}\right)_{\nu^{\prime} \nu}^{I I I, I I I} \approx & \frac{\hbar^{2} k_{\nu}^{2}}{2 m} \frac{i^{\nu^{\prime}-\nu}}{4(b-R)} \mathcal{I}_{K}(0) \int_{-b+R}^{b-R} d x\left[e^{-i k_{\nu^{\prime}} x}\right. \\
& \left.+(-1)^{\nu^{\prime}} e^{i k_{\nu^{\prime}} x}\right]\left[e^{i k_{\nu} x}+(-1)^{\nu} e^{-i k_{\nu} x}\right]
\end{aligned}
$$

where we replaced the very slowly varying function $\mathcal{I}_{K}(x)$ by its value at $x=0$, in the middle of the potential well,

$$
\mathcal{I}_{K}(0)=\int_{-1}^{1} d s \frac{\sin (s K(b-R))}{s \pi}=\frac{2}{\pi} \operatorname{Si}[K(b-R)] .
$$

Here $\operatorname{Si}(u)$ denotes the sine integral function. The integral standing in Eq. (C15) is just the same as the one in Eq. (C7) vanishing for $\nu^{\prime} \neq \nu$ and taking the value $4(b-R)$ for $\nu^{\prime}=\nu$ in the limit $V_{\infty} \rightarrow \infty$, so that we obtain

$$
\left(t_{\Pi}^{[1]}\right)_{\nu^{\prime} \nu}^{I I I, I I I}=\frac{\hbar^{2}\left(k_{\nu}^{\infty}\right)^{2}}{2 m} \mathcal{I}_{K}(0) \delta_{\nu^{\prime} \nu}
$$

and

$$
\left(t^{[1]}\right)_{\nu^{\prime} \nu}^{I I I, I I I}=\frac{\hbar^{2}\left(k_{\nu}^{\infty}\right)^{2}}{2 m}\left[\mathcal{I}_{K}(0)-1\right] \delta_{\nu^{\prime} \nu} .
$$

The corrections of various orders in the small parameter $a / \ell$ can be made explicit by making use of the asymptotic expansion of the sine integral function (see the relations $5.2 .6,5.2 .7,5.2 .8,5.2 .34,5.2 .35$ in [72]). Keeping the terms up to the order $(a / \ell)^{2}$, we find

$\mathcal{I}_{K}(0)-1 \sim-\frac{2}{\pi}\left(\frac{a \cos (K(b-R))}{\pi(b-R)}-\frac{a^{2} \sin (K(b-R))}{\pi^{2}(b-R)^{2}}\right)$.

Let us now consider the integral $\left(t^{[1]}\right)_{\nu^{\prime} \nu}^{I I, I I I}$. The term of the kernel with the Dirac-delta does not contribute and the integral reduces to

$$
\begin{aligned}
&\left(t^{[1]}\right)_{\nu^{\prime} \nu}^{I I, I I I} \\
&=-\frac{\hbar^{2}}{2 m} \frac{k_{\nu^{\prime}}^{\infty}(-i)^{\nu}}{2 \gamma c_{2}(b-R)} \\
& \times \int_{I I} d x \int_{I I I} d y \operatorname{Ai}\left(\gamma\left(-x-(-b-R)-r_{\nu^{\prime}}\right)\right) \\
& \times \partial_{x}^{2} \Pi(x-y)\left(e^{i k_{\nu} y}+(-1)^{\nu} e^{-i k_{\nu} y}\right) .
\end{aligned}
$$

Let us apply the approximation given in Eq. (C14) and make the replacement $\mathcal{I}_{K}(x) \Rightarrow \mathcal{I}_{K}(0)$, again. Then we get

$$
\begin{aligned}
\left(t^{[1]}\right)_{\nu^{\prime} \nu}^{I I, I I I} \approx & \frac{\hbar^{2} k_{\nu}^{2}}{2 m} \frac{k_{\nu^{\prime}}^{\infty}(-i)^{\nu}}{2 \gamma c_{2}(b-R)} \mathcal{I}_{K}(0) \\
& \times \int_{I I} d x \operatorname{Ai}\left(\gamma\left(-x-(-b-R)-r_{\nu^{\prime}}\right)\right) \\
& \times\left[e^{i k_{\nu} x}+(-1)^{\nu} e^{-i k_{\nu} x}\right] .
\end{aligned}
$$

As to the next we expand the integral $\int_{I I} d x \ldots$ as the function of its limits in the small parameter $2 R / \ell$, keeping the leading order term,

$$
\begin{aligned}
& \left(t^{[1]}\right)_{\nu^{\prime} \nu}^{I I, I I I} \\
& =\frac{\hbar^{2} k_{\nu}^{2}}{2 m} \frac{k_{\nu^{\prime}}^{\infty} R(-i)^{\nu}}{\gamma c_{2}(b-R)} \mathcal{I}_{K}(0) \operatorname{Ai}\left(\gamma\left(\ell+R-r_{\nu^{\prime}}\right)\right. \\
& \quad \times\left[e^{-i k_{\nu} b}+(-1)^{\nu} e^{i k_{\nu} b}\right] .
\end{aligned}
$$

In the limit $V_{\infty} \rightarrow \infty$ the argument of the Airy function goes to plus infinity, too. Therefore, the integral $\left(t^{[1]}\right)_{\nu^{\prime} \nu}^{I I, I I I}$ is exponentially suppressed in the limit $V_{\infty} \rightarrow \infty$.

In order to estimate the integral $\left(t^{[1]}\right)_{\nu^{\prime} \nu}^{I I, I I}$ we split it into the sum of the terms with the projector and the Dirac-delta, $\left(t^{[1]}\right)_{\nu^{\prime} \nu}^{I, I I}=\left(t_{\Pi}^{[1]}\right)_{\nu^{\prime} \nu}^{I I, I I}+\left(t_{D}^{[1]}\right)_{\nu^{\prime} \nu}^{I I, I I}$, where

$$
\begin{aligned}
\left(t_{\Pi}^{[1]}\right)_{\nu^{\prime} \nu}^{I I, I I}= & -\frac{\hbar^{2}}{2 m} \frac{k_{\nu^{\prime}}^{\infty} k_{\nu}^{\infty}}{\gamma^{2} c_{2}^{2}(b-R)} \\
& \times \int_{I I} d x \int_{I I} d y \operatorname{Ai}\left(\gamma\left(-x-(2 L a-R)-r_{\nu^{\prime}}\right)\right) \\
& \times \partial_{x}^{2} \Pi(x-y) \operatorname{Ai}\left(\gamma\left(-y-(b-R)-r_{\nu}\right)\right),
\end{aligned}
$$


(C23)

$$
\begin{aligned}
\left(t_{D}^{[1]}\right)_{\nu^{\prime} \nu}^{I I, I I}= & \frac{\hbar^{2}}{2 m} \frac{k_{\nu^{\prime}}^{\infty} k_{\nu}^{\infty}}{\gamma^{2} c_{2}^{2}(b-R)} \\
& \times \int_{I I} d x \int_{I I} d y \operatorname{Ai}\left(\gamma\left(-x-(b-R)-r_{\nu^{\prime}}\right)\right) \\
& \left.\times \partial_{x}^{2} \delta(x-y)\right] \operatorname{Ai}\left(\gamma\left(-y-(b-R)-r_{\nu}\right)\right) \\
= & \frac{\hbar^{2}}{2 m} \frac{k_{\nu^{\prime}}^{\infty} k_{\nu}^{\infty}}{\gamma^{2} c_{2}^{2}(b-R)} \\
& \times \int_{I I} d x \operatorname{Ai}\left(\gamma\left(-x-(b-R)-r_{\nu^{\prime}}\right)\right) \\
& \times \partial_{x}^{2} \operatorname{Ai}\left(\gamma\left(-x-(b-R)-r_{\nu}\right)\right) .
\end{aligned}
$$

In Eq. (C24) the integral over $x$ as the function of its limits can be expanded in the small parameter $2 R / \ell$, and approximated by its leading order term as

$$
\begin{aligned}
\left(t_{D}^{[1]}\right)_{\nu^{\prime} \nu}^{I I, I I} \approx & \frac{\hbar^{2}}{2 m} \frac{2 k_{\nu^{\prime}}^{\infty} k_{\nu}^{\infty} R}{\gamma^{2} c_{2}^{2}(b-R)} \operatorname{Ai}\left(\gamma\left(R-r_{\nu^{\prime}}\right)\right) \\
& \times \gamma^{2} \operatorname{Ai}^{\prime \prime}\left(\gamma\left(R-r_{\nu}\right)\right) .
\end{aligned}
$$

Since the arguments of the Airy function and its second derivative tend to $\gamma R \rightarrow+\infty$ in the limit $V_{\infty} \rightarrow \infty$, this term is exponentially suppressed and vanishes in that limit. Now let us turn to the term (C23), insert the projector in its integral form given in Eq. (7) and perform the differentiation $\partial_{x}^{2}$,

$$
\begin{aligned}
\left(t_{\Pi}^{[1]}\right)_{\nu^{\prime} \nu}^{I I, I I}= & \frac{\hbar^{2}}{2 m} \frac{k_{\nu^{\prime}}^{\infty} k_{\nu}^{\infty}}{\gamma^{2} c_{2}^{2}(b-R)} \int_{-K}^{K} \frac{d k_{x}}{2 \pi} k_{x}^{2} \\
& \times \int_{I I} d x \int_{I I} d y \operatorname{Ai}\left(\gamma\left(-x-(b-R)-r_{\nu^{\prime}}\right)\right) \\
& e^{i k_{x}(x-y)} \operatorname{Ai}\left(\gamma\left(-y-(b-R)-r_{\nu}\right)\right), \quad(\mathrm{C} 26
\end{aligned}
$$

and then replace $x$ and $y$ in the Airy function slowly varying in the narrow interval $I_{I I}$ by their values at $-b$, i.e., at the middle of the interval,

$$
\begin{aligned}
& \left(t_{\Pi \Pi}^{[1]}\right)_{\nu^{\prime} \nu}^{I I, I I} \\
& \approx \frac{\hbar^{2}}{2 m} \frac{k_{\nu^{\prime}}^{\infty} k_{\nu}^{\infty}}{\gamma^{2} c_{2}^{2}(b-R)} \operatorname{Ai}\left(\gamma\left(R-r_{\nu^{\prime}}\right)\right) \operatorname{Ai}\left(\gamma\left(R-r_{\nu}\right)\right) \\
& \quad \times \int_{-K}^{K} \frac{d k_{x}}{2 \pi} k_{x}^{2} \int_{I I} d x \int_{I I} d y e^{i k_{x}(x-y)} .
\end{aligned}
$$

The remaining integrals can be taken exactly and one finds

$$
\begin{aligned}
\left(t_{\Pi}^{[1]}\right)_{\nu^{\prime} \nu}^{I I, I I} & \\
\approx & \frac{\hbar^{2}}{2 m} \frac{k_{\nu^{\prime}}^{\infty} k_{\nu}^{\infty}}{\gamma^{2} c_{2}^{2}(b-R)} \operatorname{Ai}\left(\gamma\left(R-r_{\nu^{\prime}}\right)\right) \operatorname{Ai}\left(\gamma\left(R-r_{\nu}\right)\right) \\
& \times \frac{2}{a}\left(1-\frac{\sin (2 K R)}{2 K R}\right) .
\end{aligned}
$$

We see again that for large values of $V_{\infty}$ this contribution is exponentially suppressed due to the asymptotically large arguments of the Airy functions, so that it vanishes in the limit $V_{\infty} \rightarrow \infty$. It is worthwhile mentioning that if $V_{\infty}$ would take a finite but large value, the estimate of $\left(t^{[1]}\right)_{\nu^{\prime} \nu}^{I I, I I}$ would be of the order $\sim \gamma^{-2} a^{-1} \sim a^{-1 / 3}$. This nonanalytic dependence of the integral on the minimal length scale $a$ is a hint that the treatment of the indirect GUP effect as a perturbation may not work when the potential well is of finite depth.

Let us now turn to the integral $\left(t^{[1]}\right)_{\nu^{\prime} \nu}^{I, I I}$ given as

$$
\begin{aligned}
\left(t^{[1]}\right)_{\nu^{\prime} \nu}^{I, I I}= & -\frac{\hbar^{2}}{2 m} \frac{k_{\nu^{\prime}}^{\infty} k_{\nu}^{\infty} e^{-\zeta}}{2 \sqrt{\pi} c_{2}^{2} \gamma^{2}(b-R)(\gamma 2 R)^{1 / 4}} \\
& \times \int_{I} d x \int_{I I} d y e^{\kappa_{\nu^{\prime}}(x+b+R)} \partial_{x}^{2} \Pi(x-y) \\
& \times \operatorname{Ai}\left(\gamma\left(-y-(b-R)-r_{\nu}\right)\right) .
\end{aligned}
$$

Making the same approximation as we did for the evaluation of $\left(t_{\Pi}^{[1]}\right)_{\nu^{\prime} \nu}^{I I, I I}$, we find the analogue of Eq. (C27),

$$
\begin{aligned}
& \left(t^{[1]}\right)_{\nu^{\prime} \nu}^{I, I I} \\
& \approx \frac{\hbar^{2}}{2 m} \frac{k_{\nu^{\prime}}^{\infty} k_{\nu}^{\infty} e^{-\zeta}}{2 \sqrt{\pi} c_{2}^{2} \gamma^{2}(b-R)(\gamma 2 R)^{1 / 4}} \operatorname{Ai}\left(\gamma\left(R-r_{\nu}\right)\right) \\
& \quad \times \int_{-K}^{K} \frac{d k_{x}}{2 \pi} k_{x}^{2} \int_{I} d x e^{\kappa_{\nu^{\prime}}(x+b+R)} \int_{I I} d y e^{i k_{x}(x-y)} .
\end{aligned}
$$

The remaining threefold integral reduces to the integral

$$
\mathcal{J}_{K}=\int_{-K}^{K} \frac{d k_{x}}{2 \pi} \frac{k_{x}^{2}\left[\cos \left(k_{x} 2 R\right)-1\right]+\kappa_{\nu^{\prime}} k_{x} \sin \left(k_{x} 2 R\right)}{\kappa_{\nu^{\prime}}^{2}+k_{x}^{2}}
$$

exhibiting a finite limit for $V_{\infty} \rightarrow \infty$. Then the integral $\left(t^{[1]}\right)_{\nu^{\prime} \nu}^{I, I I}$ vanishes in the limit $V_{\infty} \rightarrow \infty$ due to the factor $e^{-\zeta}$ and the asymptotically large argument of the Airy function.

The integrals $\left(h_{t}^{[2]}+t^{[2]}\right)_{\nu^{\prime}{ }_{\nu}}^{i, j}$ can be evaluated and estimated in a rather similar manner as it was done for the integrals $\left(t^{[1]}\right)_{\nu^{\prime}{ }^{\prime} \nu}^{i, j}$. Considering the sum $\left(h_{t}^{[2]}+t^{[2]}\right)_{\nu^{\prime} \nu}^{i, j}$ instead of the integrals $\left(h_{t}^{[2]}\right)_{\nu^{\prime} \nu}^{i, j}$ and $\left(t^{[2]}\right)_{\nu^{\prime} \nu}^{i, j}$ separately has the advantage that the sum contains only the projected kinetic energy kernel. The estimates of the integrals $\left(h_{t}^{[2]}+t^{[2]}\right)_{\nu^{\prime} \nu}^{i, j}$ for $(i, j)=(I I I, I I I)$ and $(I I, I I I)$ can be obtained from the corresponding estimates of the integrals $\left(t_{\Pi}^{[1]}\right)_{\nu^{\prime} \nu}^{i, j}$ when their factor $\left(\hbar k_{\nu}^{\infty}\right)^{2} /(2 m)$ is multiplied with $(2 / 3)\left(\alpha \hbar k_{\nu}^{\infty}\right)^{2}$ that corresponds to the replacement of the ordinary kinetic energy operator in the matrix elements of $\hat{t}^{[1]}$ with the operator $(2 / 3) \alpha^{2}\left(-i \hbar \partial_{x}\right)^{4} /(2 m)$ in the matrix elements of $\hat{h}_{t}^{[2]}+$ $\hat{t}^{[2]}$. In that manner one finds that $\left(h_{t}^{[2]}+t^{[2]}\right)_{\nu^{\prime} \nu}^{I I, I I I}$ is exponentially suppressed for large values of the parameter $V_{\infty}$ and vanishes in the limit $V_{\infty} \rightarrow \infty$, while one obtains

$$
\left(h_{t}^{[2]}+t^{[2]}\right)_{\nu^{\prime} \nu}^{I I I, I I I} \sim \delta_{\nu^{\prime} \nu} \frac{2 \alpha^{2}}{3} \frac{\hbar^{4}\left(k_{\nu}^{\infty}\right)^{4}}{2 m} \mathcal{I}_{K}(0)
$$

in the limit $V_{\infty} \rightarrow \infty$. The same replacement of the ordinary kinetic energy operator in order to obtain the integrals $\left(h_{t}^{[2]}+t^{[2]}\right)_{\nu^{\prime} \nu}^{i, j}$ from the integrals $\left(t_{\Pi}^{[1]}\right)_{\nu^{\prime} \nu}^{i, j}$ with 
$(i, j)=(I I, I I)$ and $(I, I I)$ leads to the slight modification of the remaining integrals over the wave vector $k_{x}$ in Eqs. (C27) and (C30), namely to the replacement $k_{x}^{2} \Rightarrow$ const. $\times k_{x}^{4}$ in their integrands. This, however, does not alter the conclusion that the remaining integrals yield finite results, and that the integrals $\left(h_{t}^{[2]}+t^{[2]}\right)_{\nu^{\prime} \nu}^{i, j}$ with $(i, j)=(I I, I I)$ and $(I, I I)$ vanish in the limit $V_{\infty} \rightarrow \infty$. Thus we conclude that the only nonvanishing contribution to the matrix elements $\left(h_{t}^{[2]}+t^{[2]}\right)_{\nu^{\prime} \nu}$ is given by
Eq. (C32). The leading order term of the factor $\mathcal{I}_{K}(0)$ is independent of the minimal length scale $a$ and it holds $\mathcal{I}_{K}(0) \approx 1+\mathcal{O}(a / \ell)$ according to the expansion given in Eq. (C19). Therefore, the contribution in Eq. (C32) is just due to the direct GUP effect modifying the kinetic energy operator explicitly, the effect of the restriction of the kinetic energy operator to the subspace of the bandlimited wavefunctions by projection, i.e., the indirect GUP effect results in terms of third and higher orders, to be neglected in the second order of the perturbation expansion. 